\title{
Pricing, refund, and coordination optimization in a two stages supply chain of green and non-green products under hybrid production mode
}

\author{
Ata Allah Taleizadeh ${ }^{1} \cdot$ Hossein Heydarian $^{1}$
}

Received: 7 March 2017 / Accepted: 28 April 2017 / Published online: 10 July 2017

(C) Springer Science+Business Media Dordrecht 2017

\begin{abstract}
In this research, a joint pricing and refund optimization problem under cooperative and non-cooperative strategies in a two-stage supply chain consists of a supplier and a manufacturer who produces two types of product; green and non-green is developed. The features of both products in usage and functional are same but in price and environmentally aspect are different. Moreover refund policy is considered for both products that can affect on customers' decision. We explore and analyze the system performance under both noncooperative and cooperative strategies. The Stackelberg game in cooperative and Rubinstein bargaining in non-cooperative environments are applied respectively. Results show the cooperation of members increase their profits and products demand for both types of products. By increasing the value of potential demand, refund amount of green product and profits in cooperative and non-cooperative cases are increased and refund amount of non-green product is decreased. Changes of utility level sensitivity to the refund amount of green and non-green products have direct relations with refund amount and system profits, unless the utility level sensitivity to the refund amount of non-green product has contrariwise relationship with the refund amount of non-green products.
\end{abstract}

Keywords Green supply chain·Refund policy·Pricing $\cdot$ Coordination $\cdot$ Hybrid production mode

\section{Introduction}

The Green supply chain management (GSCM) has attracted significant attention of government and business communities which persuades chain members to focus on many research

Ata Allah Taleizadeh

Taleizadeh@ut.ac.ir

Hossein Heydarian

H_heydaryan@ut.ac.ir

1 School of Industrial Engineering, College of Engineering, University of Tehran, Tehran, Iran 
topics including product design, inventory management, carbon footprint measurement, refunds control, reverse logistics systems management, and coordination between the members of channels [1-8]. In this paper, we take a look on pricing and refund policies and collaboration between members in the supply chain when they cooperate for greening initiatives.

In generally, most of previous studies of GSCM work on qualitative analysis and theoretical research where less attentions are paid to quantitative aspects. One of the recent research focused on quantitative concept is work of Zhang et al. [9]. They consider a two levels green chain that contains a supplier and a producer who produces both types of green and non-green products. They investigate the pricing strategies using both cooperative and non-cooperative games between the supply chain members. Our assumptions are similar to Zhang et al. [9] by this differences that we consider the refund policy as our main contribution in this paper. Refund policy plays an important role for firms to ensure the customers that the quality level of product can satisfy their expectations [10]. The refund policy can also stimulate customer demand [11, 12]. Mukhopadhyay and Setaputra [13] indicate that around 70\% of customers consider the refund policy of retailer before making purchasing decision.

In this research we apply a refund strategy in a two levels supply chain consists of one supplier and one manufacture who produces both green and non-green products. The green products are reusable, energy efficient made with post-consumer materials, have reduced toxicity properties, manufactured with minimal environmental impact, and are delivered in eco-responsible packaging and containers. The term "green" often refers to products, services or practices that allow for economic development while conserving for future generation. A green product is one that has less of an environmental impact or is less detrimental to human health than the traditional product equivalent. We assume that supplier provides two types of raw materials for producing the green and non-green products and manufacturer sells both types of products to the customers. Selling price and refund amount of both products are determined by the manufacture. If customer is not satisfied, can return the product to manufacturer and gets back the refund amount. Moreover cooperative and non-cooperative environments between the supply chain members are considered.

In the following sections, in "Literature review" section, the relevant works in two parts; GSCM and pricing strategy are reviewed. In "Problem description" section we describe the problem and in "Computational and practical results" and "Conclusion" Sections the hybrid production mode under non-cooperative and cooperative games are discussed respectively. Finally concluding section and future research directions are provided.

\section{Literature review}

The review of research papers in this section is divided in two subsections. In the first part we focus on GSCM and in the second part, pricing strategy, refund policy, and coordination between supply chain members are reviewed.

As the first work in GSCM, Navinchandra [14] proposes the idea of green product design, for increasing products' compatibility with the environment and without compromising their functions or quality level. Vachon and Klassen [15] focus on the environmental collaboration between members in a supply chain and its influences on manufacturing and environmental performance in their survey of North American organizations. Chen and Sheu [16] study how to improve their products' recyclability by establishing a differential game model and develop 
a new dynamics analysis in terms of market competition, recycling and profit function. Ghosh and Shah [17] build game theoretical-based models to compare different structures of twolevel green supply chains. They show how channel structures can affect the selling prices, greening levels, and profits. Results showed that when the cooperation between manufacturer and the retailer is happen, green innovation could be maximized. Zhang and Liu [18] study the effects of green products on market demand of a three stages supply chain including a supplier, a producer and a retailer. They consider four models using the Stackelberg model and cooperative decision-making process. Li et al. [19] develop a dual channel GSCM model and focus on the pricing and greening strategies in both centralized and decentralized cases. Giri and Bardhan [20] study the coordination between the member of a two stages supply chain consists of a producer and a retailer where the demand of customers depends on both environmental friendliness level and retailing price of the product.

Rao and Holt [21] and Chen et al. [22] study the GSCM usages and their relationships between competitiveness and economic performance amongst a group of firms in South East Asia. Chiou et al. [23] develop a mathematical model for encouraging the firms to apply the GSCM and green innovation in order to enhance the environmental efficiency. Bowen et al. [24] study the about desirability and slow implementation of GSCM in practice. Min and Galle [25] explore the effects of "green purchasing" on packaging decisions and applying GSCM. Olugu et al. [26] develop a group of criteria to evaluate the performance of GSCM. Liang et al. [27] develop a seller-buyer supply chain model and use a group of data envelopment analysis methods to evaluate the GSCM efficiency. Cao and Zhang [28] develop the green products' utility diversity and coordination in the manufacturer- supplier green supply chain. Sheu [29] study on the negotiation insides the green supply chain members' relations that contain manufacturers and suppliers. In the next section a review on pricing, refund and coordination in supply chain is presented.

Webster and Weng [30] develop a producer-retailer supply chain model and study a refund policy and compared results with the situation that no return is allowed. Taylor [31] combines a buyback and rebate contracts to coordinate the supply chain including a producer and a wholesaler when the demand rate depend on sales effort. Yue and Raghunathan [32] focus on the information asymmetry and refund policy to coordinate the supply chains. Yao et al. [33] analyze the influences of price sensitivity factors on refund policy in a coordination problem using a mathematical model where the demand rate is stochastic and selling price-dependent. Their results indicate that refund policy can improve supply chain performance. He et al. [34] focus on a supplier-retailer supply chain contract and coordination and develop a mathematical model with stochastic and retail price- and sales effort-dependent demand. Xiao et al. [35] developed a mathematical model to coordinate the producer and the retailer of chain where refund policy, buyback contract and uncertain demand are assumed. Li et al. [11] consider the product quality as an important factor on customer behavior in online direct selling. They have developed several models to show the relations among refund policy, quality, and pricing decisions of product. Liu et al. [36] extend a Newsvendor mathematical model for a supply chain including one producer and one retailer under considering customer returns and uncertain demand. Yoo [37] develop a supplier-producer supply chain model where the supplier is risk aversion and study the relationships between decisions of quality levels and refund policy of products in a decentralized system, where the returns quantity is a proportion of market size. Moreover some related work can be found in [38-51]).

Mukhopadhyay and Setoputro [52] propose an integrated refund policy and pricing mathematical model in an online direct supply chain, and also investigate their influences on the 
customer's decisions. Ringbom and Shy [53] use partial refunds in advance booking in a single-echelon supply chain. They propose a developed method for maximizing the profit and socially optimal rates of partial refunds on customers' no-shows and cancellations. Ding and Chen [54] investigate the coordination of a three stage supply chain (supplier, manufacture, and retailer) in which product with short life cycle is sold. Su [55] develop a supply chain model of consumer refund policy and study coordination strategy in the chain. He/she study the impact of partial refund policy on the supply chain performance. Xu et al. [56] develop a two-stage supply chain including one manufacture and one retailer. The main purpose of this model is to determine of the selling price, refund and return deadline set by retailer. They assume that the return deadline strategy depends on product lifecycle and the consumer's return rate. Roy et al. [57] and Chen [58] consider a two-layer supply chain that contains a manufacturer and a retailer and use the classical newsvendor model for cooperative advertising policy. He et al. [59] develop a model to determine optimal refund policies of multi products single-period problem in supplier-retailer supply chain under uncertain demand market.

Majumder and Srinivasan [60] consider the contract leadership in a supply chain network, and derive the optimal location for the channel leader. As an extension of Choi [61], Choi [62] studies the case with the duopoly common retailer channel and finds that product (store) differentiation benefits the manufacturers (retailers) but harms the retailers (manufacturers); Choi et al. [63] investigate a CLSC which consists of a retailer, a collector, and a manufacturer, and examine the performance of CLSC under different channel leadership scenarios. Lee and Staelin [64] investigate the role of vertical strategic interaction in driving the optimal channel pricing strategy under three game scenarios. Almehdawe and Matin [65] consider a manufacturer and multiple retailers' setup and compare the efficiency of supply chain between the manufacturer-led and the retailer-led cases. Numerical analysis appears that retailer dominance generally results in higher supply chain efficiency. Gao et al. [66] explore the impact of channel power structure on the decisions and performance of a CLSC. The results show that VN structure is the most favorable for both the CLSC and consumers when the demand expansion effectiveness of collection effort is relatively low; otherwise, the CLSC with dominant retailer is most profitable. Xiao et al. [67] develop game models to explore the quoted delivery leadtime, price, and channel structure decisions for a make-to-order duopoly system under three game scenarios and discover that decentralization can increase quoted leadtime and both manufacturers may choose different channel structures under symmetric duopoly. Xu et al. [68] proposed a game model to analyze the game relationships between enterprises and consumers in the home appliance industry. Barari et al. [69] presented an evolutionary game model in which the producer manufactured green products and the retailer was responsible for attracting consumers to buy the green products. Coordination between the producer and the retailer could balance the environmental and commercial benefits. Moriarty and Moran [70] pointed out that there was inevitable channel competition between the direct channel and the retail channel. Chiang et al. [71] and Tsay and Agrawal [72] showed that channel conflict in the dual-channel supply chain threw new challenges to the manufacturer and the retailer. Yao and Liu [73] argued that in a dualchannel supply chain, the manufacturer and the retailer sold the same products, which would lead to conflict and competition between them. To mitigate channel conflict and allocate the profits properly, many scholars have explored pricing strategies between the two channels. Chiang et al. [71] showed that the adoption of a direct channel could help the manufacturer control the retailer's pricing behavior, which 
could reduce the effect of double marginalization. Fruchter and Tapiero [74] found that a manufacturer who has two distribution channels should adopt consistent pricing policies across the two channels as his optimal policy. Yao and Liu [73] discussed the pricing policies of Bertrand and Stackelberg equilibrium in a dual channel. Cattani et al. [75] analyzed consistent pricing strategies between the manufacturer and the retailer. Specifically, the manufacturer and the retailer agreed on the results when the wholesale price and sale price were set by the manufacturer to maximize his profit. Huang and Swaminathan [76] investigated optimal pricing strategies when a firm distributed a product through online and traditional channels. Dan et al. [77] examined pricing policies in a dual-channel supply chain in which the retailer provides retail service. Results showed that the pricing strategies of the channel members were greatly influenced by retail services. Martín-Herran and Taboubi [78] studied pricing decisions and coordination in distribution channels from a dynamic perspective under centralized and decentralized models. However, the above papers did not consider the situation in a green supply chain management; therefore, a new one into green supply chain management.

According to above literature review there is no research that simultaneously considers a two-level supply chain in which both green and non-green products are produced and refund policy for both products under both cooperative and non-cooperative strategies are considered. Therefore, the main contribution of this paper is modelling the refund and pricing policies in the two-level supply chain under hybrid production modes meaning producers produce two types of products.

\section{Problem description}

Consider a two stages supply chain with a supplier and a manufacture. This supply chain produces two types of products; green and non-green ones. In the aspect of usage and functional they have similar but in the price and environmental effects may exist difference between those. Also the whole sale prices of raw materials from the wholesaler to the manufacture and the production costs of non-green and green items are different.

The other issue that we consider in this paper is refund policy because the refund can occur for both products. In this paper the selling prices and refund amounts of both products directly affect the customer's decision about purchasing and returning the products. If customer is not satisfied from the products' characteristics, can return the product to the manufacturer and gives back the refund amount. In the proposed supply chain the supplier has no responsibility about the returned products.

In this supply chain, we focused on two different strategies, cooperation and competition between the supplier and the manufacture. In non-cooperative strategy we use the Stackelberg model where the supplier and the manufacturer act as the leader and follower respectively. In cooperation environment the members join together and can help the supply chain members to enhance their profits. But the main issue in a cooperative strategy in this paper is bargaining between the members in determining the raw material selling prices of both green and non-green products. In this strategy we use Rubinstein bargaining game. The structure of developed supply chain is presented in Fig. 1. The following parameters and decision variables are used in this paper to model the problem. 


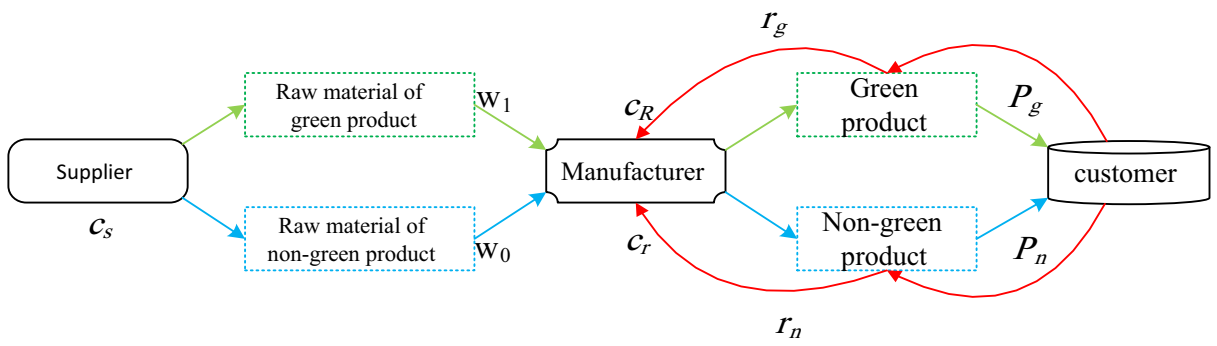

Fig. 1 Selling product and refund policy in the two-level supply chain

\section{Parameters}

$c_{R} \quad$ Production cost of green item per unit (\$/unit)

$c_{r} \quad$ Production cost of non-green item per unit (\$/unit)

$c_{S} \quad$ Supplier fixed cost for green and non-green products (\$/order)

$\varphi_{1} \quad$ Returned quantity sensitivity to the refund amount for green product

$\varphi_{2} \quad$ Returned quantity sensitivity to the refund amount for non-green product

$\tau_{1} \quad$ Returned quantity sensitivity to the selling price of green product

$\tau_{2} \quad$ Returned quantity sensitivity to the selling price of non-green product

$\phi_{1} \quad$ Independent returned quantity of green product (unit)

$\phi_{2}$ Independent returned quantity of non-green product (unit)

$v \quad$ Consumer's evaluation for products

$\pi_{m} \quad$ The manufacturer's profit (\$)

$\pi_{s} \quad$ Profit of supplier (\$)

$Q \quad$ Demand quantity (unit)

$R \quad$ Return quantity (unit)

$y_{1} \quad$ Utility level sensitivity to the refund amount of green product

$y_{2} \quad$ Utility level sensitivity to the refund amount of non-green product

\section{Decision variables}

$P_{g} \quad$ Selling price of green product (\$)

$P_{n} \quad$ Selling price of non-green product (\$)

$r_{g} \quad$ Refund of green product to be paid by the manufacturer to customer (\$)

$r_{n} \quad$ Refund of non-green product to be paid by the manufacturer to customer (\$)

$w_{1} \quad$ Price of raw material of green product (\$)

$w_{0} \quad$ Price of raw material of non-green product $(\$)$

Obviously we expected that $c_{R}>c_{r}, w_{1}>w_{0}, P_{g}>P_{n}$.

The main difference between green and non-green products to persuade the customer to choose one of them is the selling price, refund amount, and environmental values when there is no significant difference in functional usages. We assume that the consumer have different consumer valuation for products using Ferrer and Swaminathan [79] and Zhang et al. [9] works. In Eq. (1) we define the customer's utility for buying the green product. We assume $v$ is subject to uniform probability distribution within $[0, A]$. Equation (2) shows the customer valuation for non-green 
product and we define $\lambda \nu,(0<\lambda<1)$. In Eqs. (1) and (2) we consider the selling price and refund amount as important factors of a product that can influence on the utility functions. $y_{1}$ and $y_{2}$ are the return sensitivity of the utility level that indicates the effect of refund amount on the utility functions for green and non-green products.

$v$ is announced by consumer, but the selling prices, refund amounts, and raw material prices of each product are determined by supply chain members. When $U_{g}>U_{n}$ customers buy the green product, otherwise they would prefer non-green one.

$$
\begin{gathered}
U_{g}=\nu-P_{g}+y_{1} r_{g} \\
U_{n}=\lambda \nu-P_{n}+y_{2} r_{n}
\end{gathered}
$$

We define the return functions depending on the refund amount and the selling prices for green and non-green products as below [11];

$$
\begin{aligned}
& R_{g}=\phi_{1}+\varphi_{1} r_{g}+\tau_{1} P_{g} \\
& R_{n}=\phi_{2}+\varphi_{2} r_{n}+\tau_{2} P_{n} .
\end{aligned}
$$

In the next subsections, the described problem using both cooperative and non-cooperative games is modeled. Superscripts $N$ and $C$ denote non-cooperative and cooperative games respectively.

\section{Pricing strategy in non-cooperative environment}

In order to implement the non-cooperative strategy the Stackelberg game theory model is used where the supplier and the manufacture are assumed to be the leader and follower respectively. In the following we calculating the demand for each type of products and solve the optimization problem for obtaining the optimal selling price, refund amount, and raw material price of both types of products.

Demands of green and non-green items are closely related to the customer's utility function, and consumer choose one of the products that satisfies his/her expectations more than the another one. So to use the demand function two conditions should be considered: (1) $U_{g}>U_{n}$; (2) $U_{g} \leq U_{n}$. If $U_{g}>U_{n}$ the consumer buy the green product, so $\nu-P_{g}+y_{1} r_{g}>\lambda \nu-P_{n}+y_{2} r_{n}$ and we have $\nu>\frac{P_{g}-P_{n}+y_{2} r_{n}-y_{1} r_{g}}{(1-\lambda)}$ where $v \sim U[0, A]$. Then the demand quantity of green item can be calculated using Eq. (5). For simplicity we define $k=\frac{P_{g}-P_{n}+y_{2} r_{n}-y_{1} r_{g}}{(1-\lambda)}$.

$$
Q_{g}=1 . \int_{k}^{A} \frac{1}{A} d v=1+\frac{P_{n}-P_{g}+y_{1} r_{g}-y_{2} r_{n}}{(1-\lambda) \mathrm{A}}
$$

If $U_{g} \leq U_{n}$ the consumer buys the non-green product, so $U_{n} \geq 0$ i.e. $\frac{P_{n}-y_{2} r_{n}}{\lambda}<v<k$ and the market demand for non-green product can be obtained as below.

$$
Q_{n}=1 . \int_{P_{n}-y_{2} r_{n}}^{k} \frac{1}{\lambda} d v=\frac{\lambda P_{g}-P_{n}+y_{2} r_{n}-\lambda y_{1} r_{g}}{(1-\lambda) \lambda \mathrm{A}}
$$


Finally the profit function becomes as follows.

$$
\begin{aligned}
& \max _{w_{0}, w_{1}} \pi_{s}=\left(w_{1}-c_{s}\right) \cdot\left(1+\frac{P_{n}-P_{g}+y_{1} r_{g}-y_{2} r_{n}}{(1-\lambda) A}\right)+\left(w_{0}-c_{s}\right) \cdot\left(\frac{\lambda P_{g}-P_{n}+y_{2} r_{n}-\lambda y_{1} r_{g}}{(1-\lambda) \lambda A}\right) \\
& \text { s.t. : } \\
& \max _{P_{g}, P_{n}} \pi_{m}=\left(\begin{array}{l}
\left(P_{g}-w_{1}-c_{R}\right) \cdot\left(1+\frac{P_{n}-P_{g}+y_{1} r_{g}-y_{2} r_{n}}{(1-\lambda) A}\right)-r_{g}\left(\phi_{1}+\varphi_{1} r_{g}+\tau_{1} P_{g}\right) \\
+\left(P_{n}-w_{0}-c_{r}\right) \cdot\left(\frac{\lambda P_{g}-P_{n}+y_{2} r_{n}-\lambda y_{1} r_{g}}{(1-\lambda) \lambda A}\right)-r_{n}\left(\phi_{2}+\varphi_{2} r_{n}+\tau_{2} P_{n}\right)
\end{array}\right) \\
& w_{1}>w_{0}, P_{g}>P_{n}
\end{aligned}
$$

Theorem 1 Profit function of manufacturer in hybrid production mode is concave and also has a unique optimal solution, when meet the following conditions.

$$
\begin{aligned}
& A^{2} \tau_{1}^{2}\left(2 \lambda-\lambda^{2}-1\right)+2 A(1-\lambda)\left(\tau_{1} y_{1}+2 \varphi_{1}\right)-y_{1}^{2}>0 \\
& (1-\lambda)\left(A(1-\lambda)\left(A \tau_{1}^{2}-4 \varphi_{1}-2 \tau_{1} y_{1}\right)+y_{1}^{2}\right)<0 \\
& A^{3} \lambda(\lambda-1)\left(\tau_{1}^{2}\left(\lambda y_{1}+y_{2}\right)+4\left(\varphi_{1}+\varphi_{2}\right)+A \lambda \tau_{2}^{2}(\lambda-1)+2 \lambda \tau_{2}^{2}\left(\tau_{1} y_{1}+2 \varphi_{1}\right)+8 \lambda \varphi_{1} \tau_{1} \tau_{2}\right) \\
& +A^{2} \lambda\left(8(1-\lambda)\left(\varphi_{2} \tau_{2} y_{1}+\varphi_{1} \tau_{2} y_{2}+2 \varphi_{1} \varphi_{2}\right)+2 y_{1} y_{2} \tau_{1}\left(\tau_{2}(2-\lambda)+\tau_{1}\right)+\lambda y_{1}^{2}\left(\tau_{1}+\tau_{2}\right)^{2}\right) \\
& -2 A\left(y_{2}^{2}\left(2 \varphi_{1}+\tau_{1} y_{1}\right)+y_{1}^{2} \lambda\left(2 \varphi_{2}+y_{2}\left(\tau_{1}+\tau_{2}\right)\right)\right)+y_{2}^{2}\left(y_{1}^{2}+A^{2} \tau_{1}^{2}\right)>0
\end{aligned}
$$

If the above conditions are not met simultaneously, using optimization software the problem should be solved.

Proof See Appendix A.

According to above theorem, the optimal selling price, refund amount, and raw material price can be obtained using the first order derivatives of profit function with respect to the decision variables as presented in Eqs. (8) to (12).

$$
P_{g}^{N}=\frac{1}{K}\left[\begin{array}{l}
\lambda A^{3}(1-\lambda)\left(\lambda \tau_{2}^{2}\left(c_{r}+w_{0}-w_{1}-c_{R}\right)\left(2 \varphi_{1}+\tau_{1} y_{1}\right)+4 \varphi_{1}\left(2 \varphi_{2}+\tau_{2} y_{2}+\phi_{2} \lambda \tau_{2}\right)\right. \\
\left.+\lambda \tau_{2}^{2} y_{1}+4 \phi_{1} \varphi_{2} \tau_{1}+\tau_{1} \tau_{2}\left(2 \phi_{1} y_{2}-\phi_{2} y_{1}\right)\right)+\left(c_{R}+w_{1}\right)\left(A y_{2}^{2}\left(y_{1} \tau_{1}+2 \varphi_{1}\right)+y_{1}^{2} y_{2}^{2}\right) \\
+A^{2}\left(\lambda ( c _ { r } + w _ { 0 } - w _ { 1 } - c _ { R } ) \left(\tau_{2} y_{1}\left(\lambda \tau_{2} y_{1}+\tau_{1} y_{2}\right)+y_{2}^{2}\left(2 \varphi_{1}+\phi_{1} \tau_{1}\right)\right.\right. \\
-A y_{1} y_{2}^{2} \phi_{1}+\lambda(\lambda-1)\left(c_{R}+w_{1}\right)\left(\tau_{1} \tau_{2}\left(y_{1} y_{2}+8\right)+6 \varphi_{1} \tau_{2} y_{2}+2 \varphi_{2} \tau_{1} y_{1}\right) \\
\left.-2\left(\lambda \varphi_{1} \tau_{2} y_{2}-\varphi_{2} \tau_{1} y_{1}\right)\right)+\lambda y_{1} y_{2}\left(\phi_{1} \tau_{2}(2-\lambda)+\phi_{2} \tau_{1}\right)+4 \phi_{1} \varphi_{2} \lambda y_{1}(1-\lambda) \\
\left.+\lambda^{2} y_{1}^{2}\left(2 \varphi_{2}+\phi_{2} \tau_{2}\right)\right)+A\left(\lambda y_{1}^{2}\left(2\left(c_{R}+w_{1}\right)-w_{0}-c_{r}\right)\left(2 \varphi_{2}+y_{2} \tau_{2}\right)-\varphi_{2} \lambda y_{1}^{2} y_{2}\right)
\end{array}\right]
$$

$$
P_{n}^{N}=\frac{1}{K}\left[\begin{array}{l}
\lambda A^{3}(\lambda-1)\left(8 \lambda \varphi_{1} \varphi_{2}-\tau_{1}^{2}\left(2 \varphi_{2}+\tau_{2} y_{2}\right)\left(c_{r}+w_{0}-\lambda\left(c_{R}+w_{1}\right)\right)+\lambda A^{2}\left(2 \varphi_{2} \tau_{1} y_{1}\right)\right. \\
\left.+2 \lambda\left(\tau_{1} \varphi_{2}\left(y_{1}+2 \phi_{1}\right)+\tau_{2} \varphi_{1}\left(y_{2}+2 \phi_{2}\right)\right)+\phi_{2} \tau_{1}^{2} y_{2}+\lambda \tau_{1} \tau_{2}\left(\phi_{2} y_{1}+\phi_{1} y_{2}\right)\right) \\
+A^{2} \lambda \tau_{2} y_{2}\left((\lambda-2)\left(w_{0}+c_{r}\right)+\lambda\left(c_{R}+w_{1}\right)\right)\left(2 \varphi_{1}+y_{1}\right)+A^{2}\left(\lambda y_{2}^{2}\left(\phi_{1} \tau_{1}+2 \varphi_{1}\right)\right. \\
\left.+\lambda^{2} y_{1}^{2}\left(\phi_{2} \tau_{2}-2 \varphi_{2}\right)+y_{2} \lambda\left(4 \phi_{2} \varphi_{1}(1-\lambda)+\phi_{2} \tau_{1} \tau_{2}(2-\lambda)+\phi_{1} \lambda \tau_{2} y_{1}\right)\right) \\
+\left(c_{r}+w_{0}\right)\left(-y_{1}^{2} y_{2}^{2}+A\left(\lambda y_{1}^{2}\left(y_{2} \tau_{2}+2 \varphi_{2}\right)+2 y_{2}^{2}\left(y_{1} \tau_{1}+2 \varphi_{1}\right)\right)\right. \\
\left.+A^{2}\left(\tau_{1}^{2} y_{2}^{2}+(3 \lambda-2)\left(2 \varphi_{2} \lambda \tau_{1} y_{1}\right)+(\lambda-1) 8 \lambda \varphi_{1} \varphi_{2}\right)\right)+A^{4} \phi_{2} \lambda^{2} \tau_{1}^{2} \tau_{2}(\lambda-1)^{2} \\
-A \lambda y_{1} y_{2}\left(y_{1} \phi_{2}+y_{2} \phi_{1}\right)-\lambda\left(c_{R}+w_{1}\right)\left(A y_{2}^{2}\left(y_{1} \tau_{1}+2 \varphi_{1}\right)+\lambda A^{2}\left(y_{2}^{2} \tau_{1}^{2}\right)\right)
\end{array}\right]
$$




$$
\begin{gathered}
r_{g}^{N}=\frac{1}{K}\left[\begin{array}{l}
y_{2}^{2}\left(\left(w_{1}+c_{R}\right)\left(y_{1}+A \tau_{1}\right)+A\left(A \tau_{1}-y_{1}+2 \phi_{1}\right)\right)+\lambda y_{1} y_{2} A\left(\tau _ { 2 } \left(3\left(c_{r}+w_{0}\right)\right.\right. \\
\left.\left.-2\left(w_{1}+c_{R}\right)-\lambda A\right)+2 \phi_{2}\right)+\lambda \tau_{1} \tau_{2} A^{2} y_{2}\left(3 \lambda\left(c_{R}+w_{1}\right)-c_{r}-w_{0}-2 w_{1}\right) \\
+A \lambda\left(w_{0}+c_{r}-w_{1}-c_{R}\right)\left(4 \varphi_{2} y_{1}-A \tau_{2}^{2}\left(y_{1}-A \lambda \tau_{1}(\lambda-1)\right)\right) \\
+A^{3}(\lambda-1)\left(\lambda^{2} \tau_{2}^{2}\left(y_{1}+2 \phi_{1}\right)+2 \lambda \tau_{1}\left(\tau_{2}\left(\lambda \phi_{2}+y_{2}\right)+2 \varphi_{2}\right)\right)+A^{4} \lambda^{2} \tau_{1} \tau_{2}^{2}(\lambda-1)^{2} \\
+\lambda A^{2}\left(4 \varphi_{2}(\lambda-1)\left(\tau_{1}\left(w_{1}+c_{R}\right)-y_{1}-2 \phi_{1}\right)+2 \tau_{2} y_{2}\left(-2 \phi_{1}(1-\lambda)+y_{1}-2 c_{R} \tau_{1}\right)\right)
\end{array}\right] \\
r_{n}^{N}=\frac{1}{K}\left[\begin{array}{l}
y_{1}^{2}\left(y_{2}\left(c_{r}+w_{0}\right)+\lambda A\left(-y_{2}+\tau_{2}\left(\lambda A+w_{0}+c_{r}\right)+2 \phi_{2}\right)\right) \\
+A^{3} \lambda(1-\lambda)\left(\tau_{1} \tau_{2}\left(\tau_{1}\left(c_{r}+w_{0}-\lambda\left(c_{R}+w_{1}\right)\right)-\lambda\left(y_{1}+2 \phi_{1}\right)\right)+2 \phi_{2} \tau_{1}^{2}-4 \varphi_{1} \lambda \tau_{2}\right) \\
+4(\lambda-1)\left(\varphi_{1} \tau_{2}\left(c_{r}+w_{0}\right)+\phi_{2} \lambda\left(2 \varphi_{1}+\tau_{1} y_{1}\right)+\lambda \tau_{1} y_{1} y_{2}\right) \\
y_{2} A\left(y_{1} \tau_{1}\left(3 \lambda\left(c_{R}+w_{1}\right)-2\left(c_{r}+w_{0}\right)\right)-4 \varphi_{1}\left(c_{r}+w_{0}+\lambda\left(w_{1}+c_{R}\right)+2 \lambda y_{1} \phi_{1}\right)\right)
\end{array}\right] \\
K= \\
+A^{2}\left(8 \lambda(\lambda-1)\left(2 \varphi_{1} \varphi_{2}+\tau_{1} y_{1} \varphi_{2}+\varphi_{1} \tau_{2} y_{2}\right)+2 \lambda \tau_{1} \tau_{2} y_{1} y_{2}(2-\lambda)\right. \\
\left.+\lambda^{2} \tau_{2}^{2} y_{1}^{2}+\tau_{1}^{2} y_{2}^{2}\right)+2 \lambda A^{3}(\lambda-1)\left(\lambda \tau_{2}^{2}\left(y_{1} \tau_{1}+2 \varphi_{1}\right)+\tau_{1}^{2}\left(2 \varphi_{2}+\tau_{2} y_{2}\right)\right) \\
-A\left(2 \lambda y_{1}^{2}\left(\tau_{2} y_{2}+2 \varphi_{2}\right)+2 y_{2}^{2}\left(\tau_{1} y_{1}+2 \varphi_{1}\right)\right)+y_{1}^{2} y_{2}^{2}+\left(A^{2} \tau_{1} \tau_{2} \lambda(\lambda-1)\right)^{2}
\end{gathered}
$$

By replacing Eqs. (8) to (12) in supplier's profit function presented in Eqs. (7) one can obtain the selling price of optimal raw material for both green and non-green items.

$$
\begin{aligned}
w_{1}^{N}= & \frac{\left(c_{s}-c_{R}\right)}{2} \\
& +\frac{A\left(\begin{array}{l}
-A \tau_{1}^{2}\left(\varphi_{2}+\tau_{2} y_{2}\right)-A \tau_{2}^{2} \lambda^{2}\left(\varphi_{1}+\tau_{1} y_{1}\right)+\tau_{1} \tau_{2} y_{2}\left(y_{1}-\phi_{1}\right) \\
+2 \varphi_{1}\left(\tau_{2} y_{2}+\varphi_{2}\right)-\phi_{2} \lambda \tau_{2}\left(\tau_{1} y_{1}+\varphi_{1}\right)-\varphi_{2} \tau_{1}\left(\phi_{1}-y_{1}\right)
\end{array}\right)-\phi_{1} y_{1}\left(\varphi_{2}+\tau_{2} y_{2}\right)}{4\left(\varphi_{1} \varphi_{2}+\varphi_{2} \tau_{1} y_{1}+\varphi_{1} \tau_{2} y_{2}+\tau_{1} \tau_{2} y_{1} y_{2}\right)} \\
w_{0}^{N}= & \frac{\left(c_{s}-c_{r}\right)}{2} \\
& +\frac{A \lambda\left(\begin{array}{l}
-A \lambda\left(\tau_{1}^{2}\left(\varphi_{2}+\tau_{2} y_{2}\right)+\tau_{2}^{2}\left(\varphi_{1}+\tau_{1} y_{1}\right)\right)+\varphi_{1}\left(2 \varphi_{2}+\tau_{2} y_{2}\right) \\
+\varphi_{2} \tau_{1}\left(-\phi_{1}+2 y_{1}\right)+\tau_{1} \tau_{2} y_{2}\left(y_{1}-\phi_{1}\right)-\phi_{2} \tau_{2}\left(\varphi_{1}+\tau_{1} y_{1}\right)
\end{array}\right)-\phi_{2} y_{2}\left(y_{1} \tau_{1}+\varphi_{1}\right)}{4\left(\varphi_{1} \varphi_{2}+\varphi_{2} \tau_{1} y_{1}+\varphi_{1} \tau_{2} y_{2}+\tau_{1} \tau_{2} y_{1} y_{2}\right)}
\end{aligned}
$$

\section{Pricing strategy in cooperative case}

In this section we model and optimize the problem using a cooperative strategy. In this strategy, supply chain members join together with the objective of increasing their profits. The bargaining between supplier and manufacturer happen when they decide to determine the raw material prices for two products. For solving this problem we use Rubinstein bargaining model.

If the supplier and manufacturer enter into the cooperation in hybrid production mode, they will conduct joint decision-making in the principal of maximizing supply chain profit where the profit of supply chain is presented in Eq. (15). 


$$
\begin{gathered}
\max _{P_{g}, P_{n}} \pi=\left(P_{g}-c_{s}-c_{R}\right) \cdot\left(1+\frac{P_{n}-P_{g}+y_{1} r_{g}-y_{2} r_{n}}{(1-\lambda) A}\right)-r_{g}\left(\Phi_{1}+\varphi_{1} r_{g}+\tau_{1} P_{g}\right) \\
+\left(P_{n}-c_{s}-c_{r}\right) \cdot\left(\frac{\lambda P_{g}-P_{n}+y_{2} r_{n}-\lambda y_{1} r_{g}}{(1-\lambda) \lambda A}\right)-r_{n}\left(\Phi_{2}+\varphi_{2} r_{n}\right)+\tau_{2} P_{n}
\end{gathered}
$$

Theorem 2 Profit function in hybrid production mode and cooperative strategy is concave and also has a unique optimal solution, when the following conditions are met.

$$
\begin{aligned}
& A^{2} \tau_{1}^{2}\left(2 \lambda-\lambda^{2}-1\right)+2 A(1-\lambda)\left(\tau_{1} y_{1}+2 \varphi_{1}\right)-y_{1}^{2}>0 \\
& (1-\lambda)\left(A(1-\lambda)\left(A \tau_{1}^{2}-4 \varphi_{1}-2 \tau_{1} y_{1}\right)+y_{1}^{2}\right)<0 \\
& A^{3} \lambda(\lambda-1)\left(\tau_{1}^{2}\left(\lambda y_{1}+y_{2}\right)+4\left(\varphi_{1}+\varphi_{2}\right)+A \lambda \tau_{2}^{2}(\lambda-1)+2 \lambda \tau_{2}^{2}\left(\tau_{1} y_{1}+2 \varphi_{1}\right)+8 \lambda \varphi_{1} \tau_{1} \tau_{2}\right) \\
& +A^{2} \lambda\left(8(1-\lambda)\left(\varphi_{2} \tau_{2} y_{1}+\varphi_{1} \tau_{2} y_{2}+2 \varphi_{1} \varphi_{2}\right)+2 y_{1} y_{2} \tau_{1}\left(\tau_{2}(2-\lambda)+\tau_{1}\right)+\lambda y_{1}^{2}\left(\tau_{1}+\tau_{2}\right)^{2}\right) \\
& -2 A\left(y_{2}^{2}\left(2 \varphi_{1}+\tau_{1} y_{1}\right)+y_{1}^{2} \lambda\left(2 \varphi_{2}+y_{2}\left(\tau_{1}+\tau_{2}\right)\right)\right)+y_{2}^{2}\left(y_{1}^{2}+A^{2} \tau_{1}^{2}\right)>0
\end{aligned}
$$

If the above conditions are met, by setting the first order derivatives of profit functions with respect to the decision variables, one can obtain the optimal values as shown in Eq. (16) to (20).

$$
\begin{aligned}
& P_{g}^{C}=\frac{1}{K}\left[\begin{array}{l}
\lambda A^{3}(1-\lambda)\left(\lambda \tau_{2}^{2}\left(c_{r}-c_{R}\right)\left(2 \varphi_{1}+\tau_{1} y_{1}\right)+4 \varphi_{1}\left(2 \varphi_{2}+\tau_{2} y_{2}+\phi_{2} \lambda \tau_{2}\right)\right. \\
\left.+\lambda \tau_{2}^{2} y_{1}+4 \phi_{1} \varphi_{2} \tau_{1}+\tau_{1} \tau_{2}\left(2 \phi_{1} y_{2}-\phi_{2} y_{1}\right)\right)+y_{2}^{2}\left(2 \varphi_{1}+\phi_{1} \tau_{1}\right) \\
+A^{2}\left(\lambda ( c _ { r } - c _ { R } ) \left(\tau_{2} y_{1}\left(\lambda \tau_{2} y_{1}+\tau_{1} y_{2}\right)+\left(c_{R}+c_{s}\right)\left(A y_{2}^{2}\left(y_{1} \tau_{1}+2 \varphi_{1}\right)+y_{1}^{2} y_{2}^{2}\right)\right.\right. \\
-A y_{1} y_{2}^{2} \phi_{1}+\lambda(\lambda-1)\left(c_{R}+c_{s}\right)\left(\tau_{1} \tau_{2}\left(y_{1} y_{2}+8\right)+6 \varphi_{1} \tau_{2} y_{2}+2 \varphi_{2} \tau_{1} y_{1}\right) \\
\left.-2\left(\lambda \varphi_{1} \tau_{2} y_{2}-\varphi_{2} \tau_{1} y_{1}\right)\right)+\lambda y_{1} y_{2}\left(\phi_{1} \tau_{2}(2-\lambda)+\phi_{2} \tau_{1}\right)+4 \phi_{1} \varphi_{2} \lambda y_{1}(1-\lambda) \\
\left.+\lambda^{2} y_{1}^{2}\left(2 \varphi_{2}+\phi_{2} \tau_{2}\right)\right)+A\left(\lambda y_{1}^{2}\left(2 c_{R}+c_{s}-c_{r}\right)\left(2 \varphi_{2}+y_{2} \tau_{2}\right)-\varphi_{2} \lambda y_{1}^{2} y_{2}\right)
\end{array}\right] \\
& P_{n}^{C}=\frac{1}{K}\left[\begin{array}{l}
\lambda A^{3}(\lambda-1)\left(8 \lambda \varphi_{1} \varphi_{2}-\tau_{1}^{2}\left(2 \varphi_{2}+\tau_{2} y_{2}\right)\left(c_{r}+c_{s}-\lambda\left(c_{R}+c_{s}\right)\right)+\lambda A^{2}\left(2 \varphi_{2} \tau_{1} y_{1}\right)\right. \\
\left.+2 \lambda\left(\tau_{1} \varphi_{2}\left(y_{1}+2 \phi_{1}\right)+\tau_{2} \varphi_{1}\left(y_{2}+2 \phi_{2}\right)\right)+\phi_{2} \tau_{1}^{2} y_{2}+\lambda \tau_{1} \tau_{2}\left(\phi_{2} y_{1}+\phi_{1} y_{2}\right)\right) \\
+A^{2} \lambda \tau_{2} y_{2}\left((\lambda-2)\left(c_{s}+c_{r}\right)+\lambda\left(c_{R}+c_{s}\right)\right)\left(2 \varphi_{1}+y_{1}\right)+A^{2}\left(\lambda y_{2}^{2}\left(\phi_{1} \tau_{1}+2 \varphi_{1}\right)\right. \\
\left.+\lambda^{2} y_{1}^{2}\left(\phi_{2} \tau_{2}-2 \varphi_{2}\right)+y_{2} \lambda\left(4 \phi_{2} \varphi_{1}(1-\lambda)+\phi_{2} \tau_{1} \tau_{2}(2-\lambda)+\phi_{1} \lambda \tau_{2} y_{1}\right)\right) \\
+\left(c_{r}+c_{s}\right)\left(-y_{1}^{2} y_{2}^{2}+A\left(\lambda y_{1}^{2}\left(y_{2} \tau_{2}+2 \varphi_{2}\right)+2 y_{2}^{2}\left(y_{1} \tau_{1}+2 \varphi_{1}\right)\right)\right. \\
\left.+A^{2}\left(\tau_{1}^{2} y_{2}^{2}+(3 \lambda-2)\left(2 \varphi_{2} \lambda \tau_{1} y_{1}\right)+(\lambda-1) 8 \lambda \varphi_{1} \varphi_{2}\right)\right)+A^{4} \phi_{2} \lambda^{2} \tau_{1}^{2} \tau_{2}(\lambda-1)^{2} \\
-A \lambda y_{1} y_{2}\left(y_{1} \phi_{2}+y_{2} \phi_{1}\right)-\lambda\left(c_{R}+c_{s}\right)\left(A y_{2}^{2}\left(y_{1} \tau_{1}+2 \varphi_{1}\right)+\lambda A^{2}\left(y_{2}^{2} \tau_{1}^{2}\right)\right)
\end{array}\right] \\
& r_{g}^{C}=\frac{1}{K}\left[\begin{array}{l}
y_{2}^{2}\left(\left(c_{s}+c_{R}\right)\left(y_{1}+A \tau_{1}\right)+A\left(A \tau_{1}-y_{1}+2 \phi_{1}\right)\right)+\lambda y_{1} y_{2} A\left(\tau _ { 2 } \left(3\left(c_{r}+c_{s}\right)\right.\right. \\
\left.\left.-2\left(c_{s}+c_{R}\right)-\lambda A\right)+2 \phi_{2}\right)+\lambda \tau_{1} \tau_{2} A^{2} y_{2}\left(3 \lambda\left(c_{R}+c_{s}\right)-c_{r}-3 c_{s}\right) \\
+A \lambda\left(c_{r}-c_{R}\right)\left(4 \varphi_{2} y_{1}-A \tau_{2}^{2}\left(y_{1}-A \lambda \tau_{1}(\lambda-1)\right)\right)+A^{4} \lambda^{2} \tau_{1} \tau_{2}^{2}(\lambda-1)^{2} \\
+A^{3}(\lambda-1)\left(\lambda^{2} \tau_{2}^{2}\left(y_{1}+2 \phi_{1}\right)+2 \lambda \tau_{1}\left(\tau_{2}\left(\lambda \phi_{2}+y_{2}\right)+2 \varphi_{2}\right)\right) \\
+\lambda A^{2}\left(2 \tau_{2} y_{2}\left(y_{1}-2 c_{R} \tau_{1}-2 \phi_{1}(1-\lambda)\right)+4 \varphi_{2}(\lambda-1)\left(\tau_{1}\left(c_{s}+c_{R}\right)-y_{1}-2 \phi_{1}\right)\right)
\end{array}\right]
\end{aligned}
$$




$$
\begin{aligned}
r_{n}^{N}=\frac{1}{K}\left[\begin{array}{l}
y_{1}^{2}\left(y_{2}\left(c_{r}+w_{0}\right)+\lambda A\left(-y_{2}+\tau_{2}\left(\lambda A+w_{0}+c_{r}\right)+2 \phi_{2}\right)\right) \\
+A^{3} \lambda(1-\lambda)\left(\tau_{1} \tau_{2}\left(\tau_{1}\left(c_{r}+w_{0}-\lambda\left(c_{R}+w_{1}\right)\right)-\lambda\left(y_{1}+2 \phi_{1}\right)\right)+2 \phi_{2} \tau_{1}^{2}-4 \varphi_{1} \lambda \tau_{2}\right) \\
A^{2}\left(\lambda \tau _ { 1 } \tau _ { 2 } y _ { 1 } \left((3 \lambda-2)\left(c_{r}+w_{0}\right)-\lambda\left(w_{1}+c_{R}\right)+\tau_{1}^{2} y_{2}\left(-\lambda\left(c_{R}+w_{1}\right)+w_{0}+c_{r}\right)\right.\right. \\
+4(\lambda-1)\left(\varphi_{1} \tau_{2}\left(c_{r}+w_{0}\right)+\phi_{2} \lambda\left(2 \varphi_{1}+\tau_{1} y_{1}\right)+\lambda \tau_{1} y_{1} y_{2}\right) \\
y_{2} A\left(y_{1} \tau_{1}\left(3 \lambda\left(c_{R}+w_{1}\right)-2\left(c_{r}+w_{0}\right)\right)-4 \varphi_{1}\left(c_{r}+w_{0}+\lambda\left(w_{1}+c_{R}\right)+2 \lambda y_{1} \phi_{1}\right)\right)
\end{array}\right] \\
K=A^{2}\left(8 \lambda(\lambda-1)\left(2 \varphi_{1} \varphi_{2}+\tau_{1} y_{1} \varphi_{2}+\varphi_{1} \tau_{2} y_{2}\right)+2 \lambda \tau_{1} \tau_{2} y_{1} y_{2}(2-\lambda)\right. \\
\left.+\lambda^{2} \tau_{2}^{2} y_{1}^{2}+\tau_{1}^{2} y_{2}^{2}\right)+2 \lambda A^{3}(\lambda-1)\left(\lambda \tau_{2}^{2}\left(y_{1} \tau_{1}+2 \varphi_{1}\right)+\tau_{1}^{2}\left(2 \varphi_{2}+\tau_{2} y_{2}\right)\right) \\
-A\left(2 \lambda y_{1}^{2}\left(\tau_{2} y_{2}+2 \varphi_{2}\right)+2 y_{2}^{2}\left(\tau_{1} y_{1}+2 \varphi_{1}\right)\right)+y_{1}^{2} y_{2}^{2}+\left(A^{2} \tau_{1} \tau_{2} \lambda(\lambda-1)\right)^{2}
\end{aligned}
$$

Moreover Eq. (21) shows the profits of supplier and manufacturer in cooperative strategy in the hybrid production mode respectively. Cooperative in the supply chain must be caused to increase the profits of supplier and manufacturer. According to Eq. (21) the price of raw materials $\left(w_{1}, w_{0}\right)$ has an important role to determine how the supply chain profit would be allocated between the supplier and manufacturer. The supplier must determine a fair and reasonable price of raw material to satisfy the manufacturer's expectations, because determining the price of raw material leads to increase profit on both sides in the supply chain.

$$
\begin{aligned}
\pi_{s}^{C} & =\left(w_{1}-c_{s}\right)\left(Q_{g}\right)+\left(w_{0}-c_{s}\right)\left(Q_{n}\right) \\
\pi_{m}^{C} & =\left(P_{g}-c_{R}-w_{1}\right)\left(Q_{g}\right)-r_{g}\left(\phi_{1}+\varphi_{1} r_{g}+\tau_{1} P_{g}\right)+\left(P_{n}-c_{r}-w_{0}\right)\left(Q_{n}\right)-r_{n}\left(\phi_{2}+\varphi_{2} r_{n}+\tau_{2} P_{n}\right)
\end{aligned}
$$

Reasonable values of selling price, refund amount, and raw material selling price make the both sides in the supply chain sure that their profits in cooperative strategy are not less than non-cooperative cases and maximize the profit of the system. So we hope that the profit of manufacturer and supplier has increased in the cooperative strategy versus in the noncooperative strategy.

$$
\pi_{s}^{C} \geq \pi_{s}^{N}, \quad \pi_{m}^{C} \geq \pi_{m}^{N}
$$

By simplifying Eq. (22), we can obtain the minimum and maximum values of raw materials selling prices for both green and non-green products as below;

$$
\begin{gathered}
w_{1}^{\min }=\frac{\left(3 c_{s}-c_{R}\right)\left(2 \varphi_{1}+2 \tau_{1} y_{1}\right)+A \tau_{1}\left(y_{1}-\phi_{1}-A \tau_{1}\right)+2 A \varphi_{1}-\phi_{1} y_{1}}{8\left(\varphi_{1}+\tau_{1} y_{1}\right)} \\
w_{1}^{\max }=\frac{\left(5 c_{s}-3 c_{R}\right)\left(2 \varphi_{1}+2 \tau_{1} y_{1}\right)+3 A \tau_{1}\left(y_{1}-\phi_{1}-A \tau_{1}\right)+6 A \varphi_{1}-3 \phi_{1} y_{1}}{16\left(\varphi_{1}+\tau_{1} y_{1}\right)} \\
w_{0}^{\min }=\frac{\left(3 c_{s}-c_{r}\right)\left(2 \varphi_{2}+2 \tau_{2} y_{2}\right)+A \lambda \tau_{2}\left(y_{2}-\phi_{2}-A \lambda \tau_{2}\right)+2 A \lambda \varphi_{2}-\phi_{2} y_{2}}{8\left(\varphi_{2}+\tau_{2} y_{2}\right)} \\
w_{0}^{\max }=\frac{\left(5 c_{s}-3 c_{r}\right)\left(2 \varphi_{2}+2 \tau_{2} y_{2}\right)+3 A \lambda \tau_{2}\left(y_{2}-\phi_{2}-A \lambda \tau_{2}\right)+6 A \lambda \varphi_{2}-3 \phi_{2} y_{2}}{16\left(\varphi_{2}+\tau_{2} y_{2}\right)}
\end{gathered}
$$

In the other hands in Eqs. (23) to (26) the minimum and maximum amounts of ( $\left.w_{0}, w_{1}\right)$ are obtained to have a reasonable cooperative supply chain. In the cooperative game manufacture 
wants to choose the raw material selling price to be close to $\left(w_{1}^{\min }, w_{0}^{\min }\right)$ and the supplier wants to be close to $w_{1}^{\max }, w_{0}^{\max }$. For finding the best pricing strategy, the Rubinstein bargaining model is used. Rubinstein [80] proved when the infinite periodic offering game between two players happen there is a unique sub-game perfect equilibrium.

$$
\theta_{1}^{*}=\frac{1-\delta_{2}}{1-\delta_{1} \delta_{2}}
$$

where $\delta_{1}$ and $\delta_{2}$ represent the fixed discounting factors such as members position in market, risk- taking and etc. for manufacturer and supplier respectively. If both $\delta_{1}$ and $\delta_{2}$ are determined then for both green and non-green products the following Equation can be used.

$$
\frac{w^{\max }-w^{C}}{w^{C}-w^{\min }}=\frac{\theta_{1}^{*}}{1-\theta_{1}^{*}}=\frac{1-\delta_{2}}{\delta_{2}\left(1-\delta_{1}\right)}
$$

So, according to Eq. (28) one can obtain the optimal selling price of raw material for green and non-green products as below;

$$
\begin{gathered}
w_{1}{ }^{C}=\frac{\left(1-\Delta_{2}\right) w_{1}{ }^{\min }+\Delta_{2}\left(1-\Delta_{1}\right) w_{1}{ }^{\max }}{1-\Delta_{1} \Delta_{2}} \\
w_{0}{ }^{C}=\frac{\left(1-\nabla_{2}\right) w_{0}{ }^{\min }+\nabla_{2}\left(1-\nabla_{1}\right) w_{0}{ }^{\max }}{1-\nabla_{1} \nabla_{2}}
\end{gathered}
$$

where $\Delta_{i}, \nabla_{i}, \delta_{1}$ and $\delta_{2}$ are fixed discounting factors of supply chain member, and $i=1$ and $i=2$ refers to manufacturer and supplier respectively.

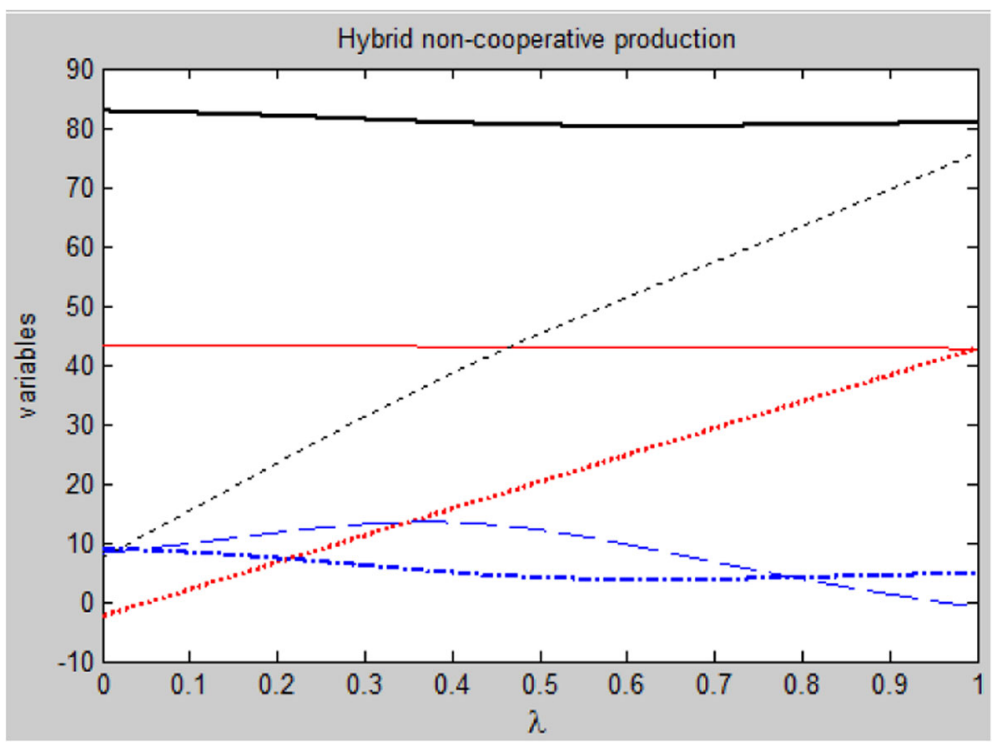

Fig. 2 The effect of $\lambda$ on variables in non-cooperative game 


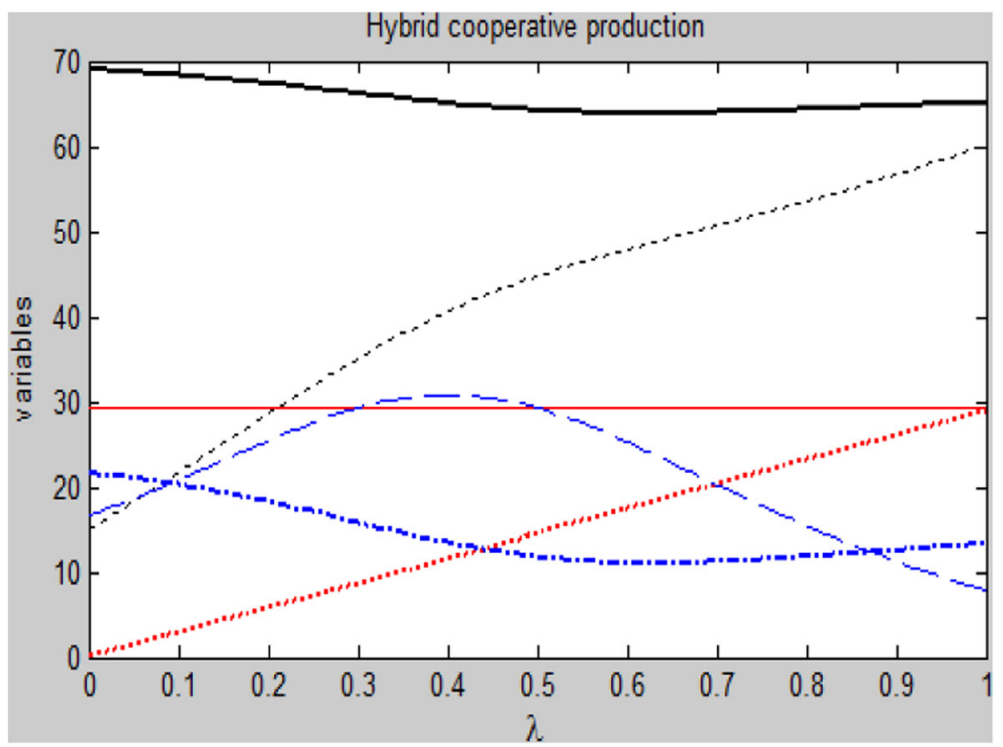

Fig. 3 The effect of $\lambda$ on variables in cooperative game

\section{Computational and practical results}

Consider a two stages supply chain with a supplier and a manufacture. This supply chain produces two types of products; green and non-green ones. In the aspect of usage and functional they have similar but in the price and environmental effects may exist difference between those. Also the whole sale prices of raw materials from the wholesaler to the manufacture and the production costs of non-green and green items are different.

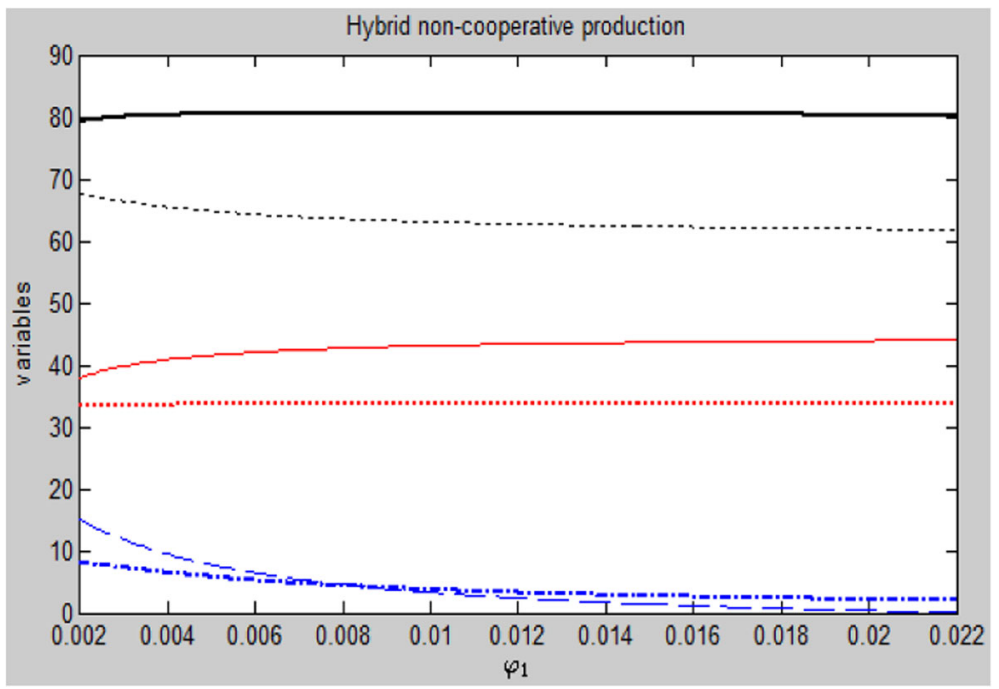

Fig. 4 The effect of $\varphi_{1}$ on variables in non-cooperative game 


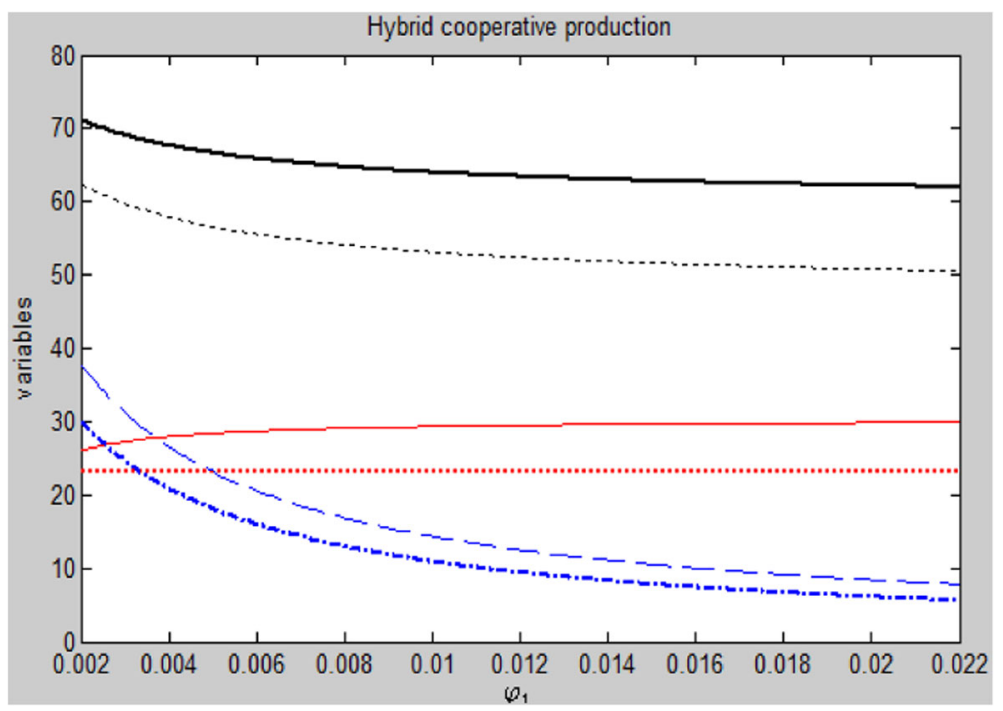

Fig. 5 The effect of $\varphi_{1}$ on variables in cooperative game

For analyzing the effect of refund policy and hybrid production mode in the proposed twolevel supply chain in both cooperative and non-cooperative strategies between members of the supply chain we use some numerical examples. We assume $c_{s}=5 \$$ item, $c_{R}=15 \$ /$ item, $c_{r}=$ $10 \$ /$ item, $A=100, \lambda=0.8, y_{1}=y_{2}=0.9$ and refund parameters as $\phi_{1}=0.003, \phi_{2}=$ $0.002, \varphi_{1}=0.009, \varphi_{2}=0.004, \tau_{2}=0.0009, \tau_{1}=0.0007$.

\section{The effect of parameters on profits and decision variables}

The influences of parameters on decision variables, finished product selling price, raw material selling price, and refund amount for both, green and non-green products under cooperative and

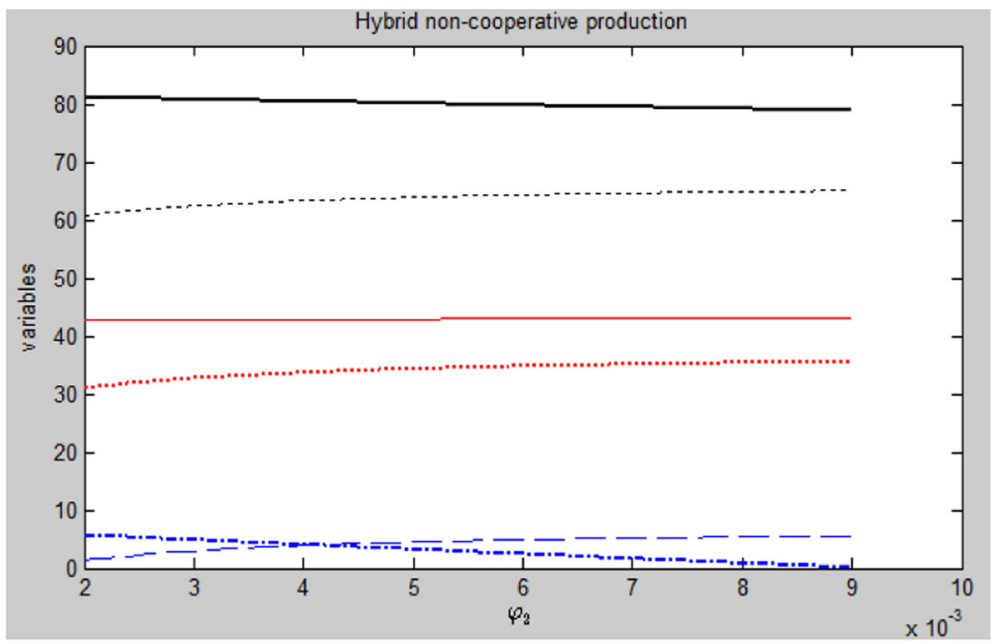

Fig. 6 The effect of $\varphi_{2}$ on variables in non-cooperative game 


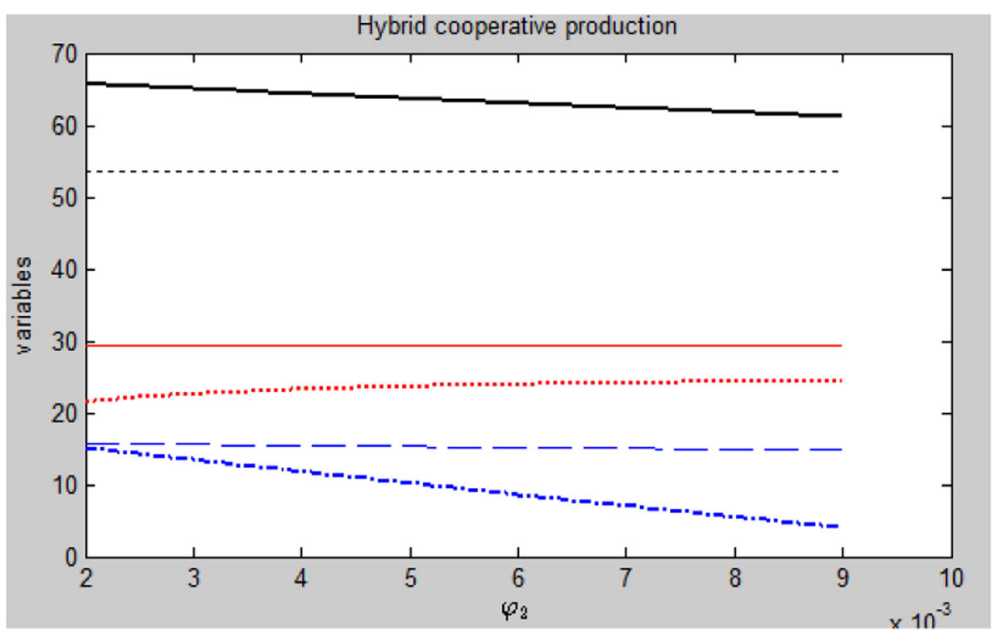

Fig. 7 The effect of $\varphi_{2}$ on variables in cooperative game

non-cooperative games are investigated. From Figs. 2, 3, 4, 5, 6, 7, 8, 9, 10, 11, 12, 13, 14, and 15 one can observe that ever $w_{1}>w_{0}$ and $P_{g}>P_{n}$, but the refund amount of green and nongreen products are fluctuated. In Figs. 2 and 3 the effects of $\lambda$ on decision variables under cooperative and non-cooperative strategies are presented. According to these figures the finished product selling price and raw material wholesale price of green product are not sensitive to the change of $\lambda$ while non-green product has a direct relationship to the changes.

$\varphi_{1}$ and $\varphi_{2}$ almost have the same effects on decision variables as presented in Figs. 4, 5, 6, and 7. The decision variables are slightly sensitive with respect to the changes of $\varphi_{1}$ and $\varphi_{2}$. Figs. 8, 9, 10, and 11 show the effects of $\tau_{1}$ and $\tau_{2}$ on decision variables. It is clear that none of decision variables are sensitive respect to the changes of $\tau_{1}$ and $\tau_{2}$.

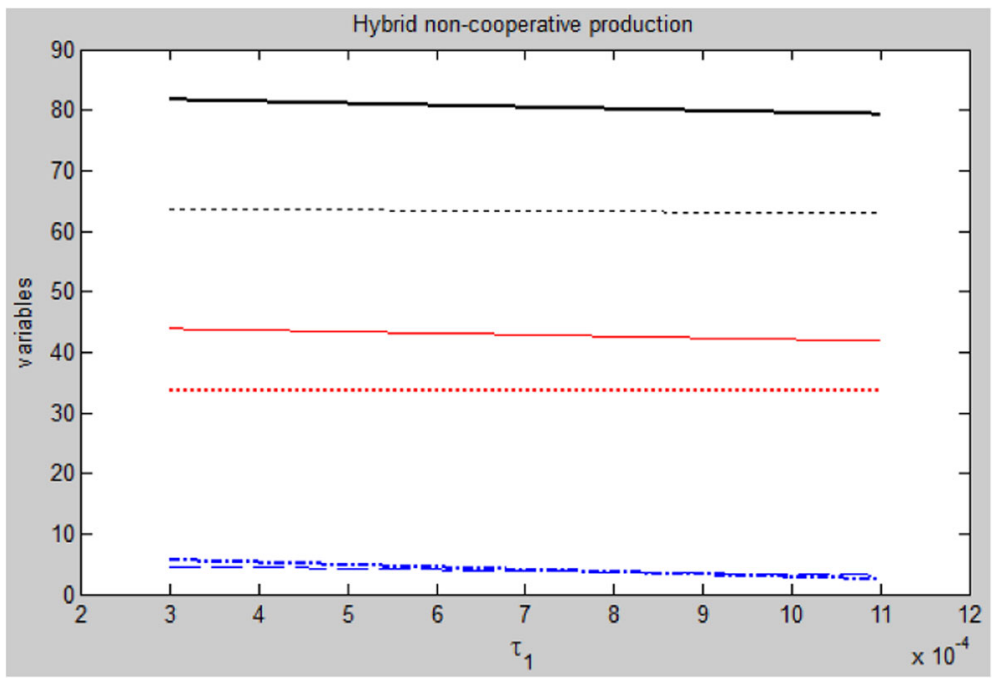

Fig. 8 The effect of $\tau_{1}$ on variables in non-cooperative game 


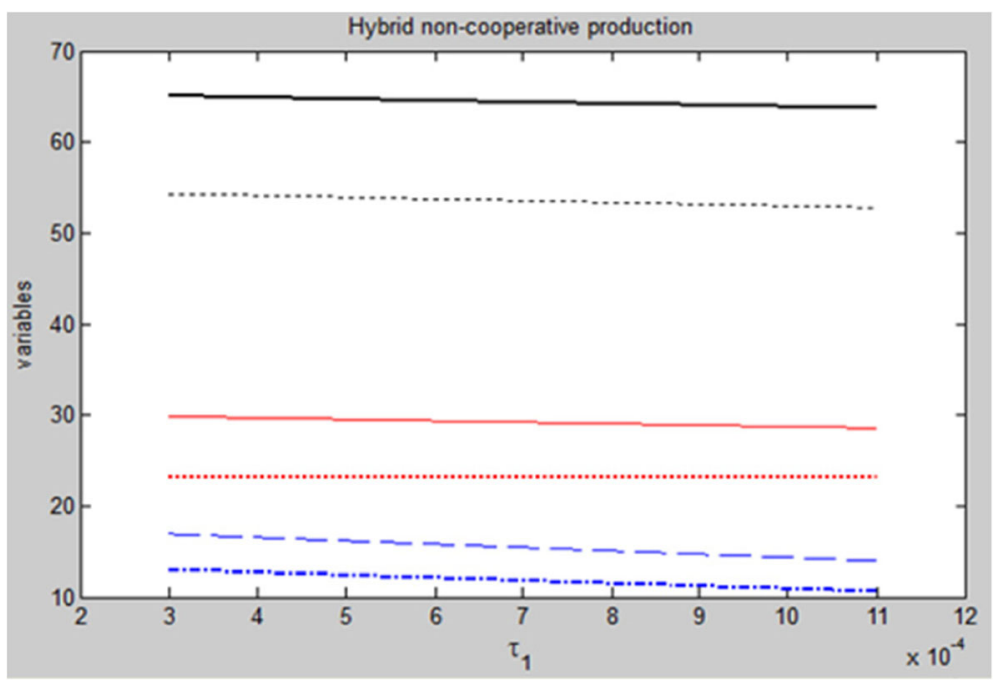

Fig. 9 The effect of $\tau_{1}$ on variables in cooperative game

As presented in Figs. 12 and 13, $y_{1}$ has direct effect on refund amount especially in cooperative strategy. Fig. 14 and 15 demonstrate the effects of $y_{2}$ on decision variables. When $y_{2}<6.2$ in non-cooperative and when $y_{2}<5.5$ in cooperative game, the system does not produce the green product and just produce non green one.

\section{Effect of parameters on demands}

The influences of parameters on demands of green and non-green products in both cooperative and non-cooperative situations are studied in this section. Figs. 16, 17, 18, 19, 20, 21, and 22 demonstrate the influences of the parameters on decisions from which it is clear that the

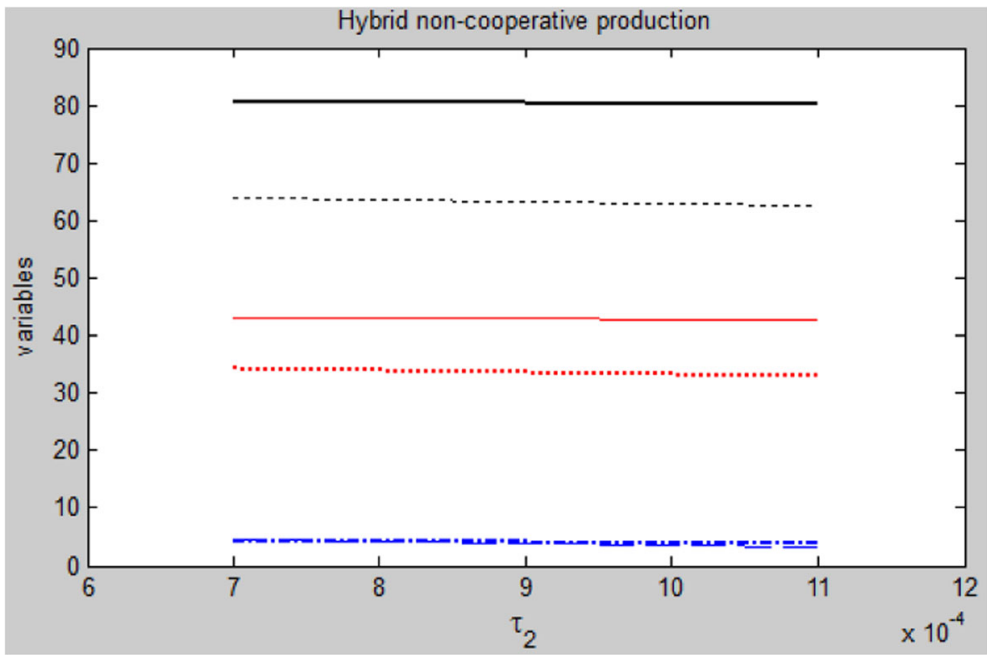

Fig. 10 The effect of $\tau_{2}$ on variables in non-cooperative game 


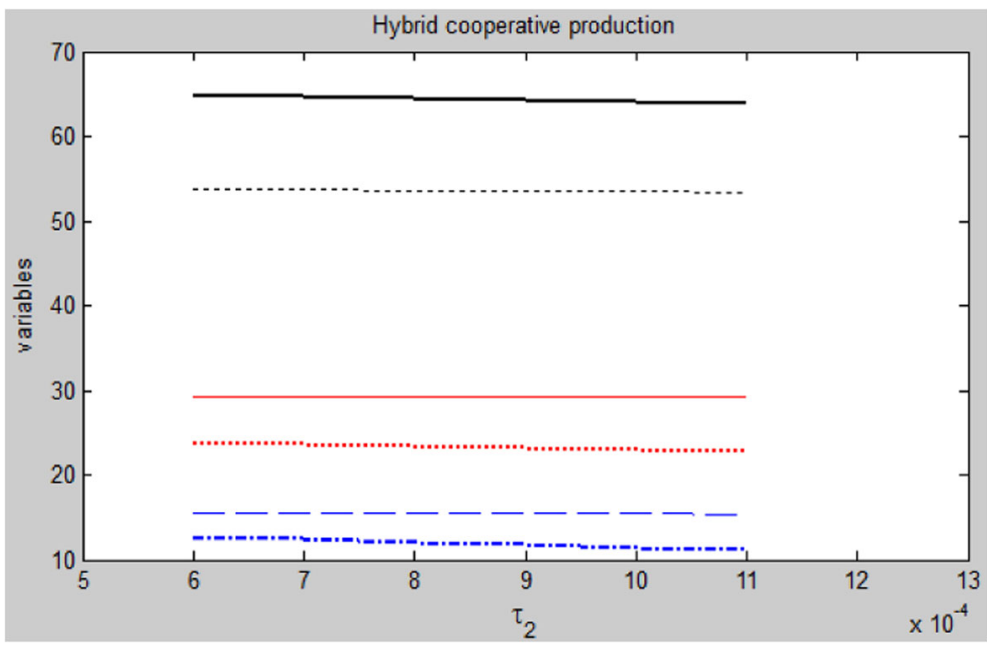

Fig. 11 The effect of $\tau_{2}$ on variables in cooperative game

demands amounts in cooperative situation for both products are more than demands in noncooperative one. Figure 16 demonstrates that the $\lambda$ influences on demands, it shows that the green product has a convex behavior and the non-green product has a concave behavior. Indeed when $\lambda$ increases the demand quantity of non-green products first increase and then decrease while the demand quantity of green products first decrease and then increase.

Figure 17 and 18 show the effects of $\varphi_{1}$ and $\varphi_{2}$, where in Fig. 17 the changes have an exponential pattern and by increasing of $\varphi_{1}$ the demand quantity of green products increase while the demand quantity of non-green one decrease. But the patterns of changes with respect to $\varphi_{2}$ are completely different and have linear form. By increase of $\varphi_{2}$, the demands quantity of green products decrease but the demand

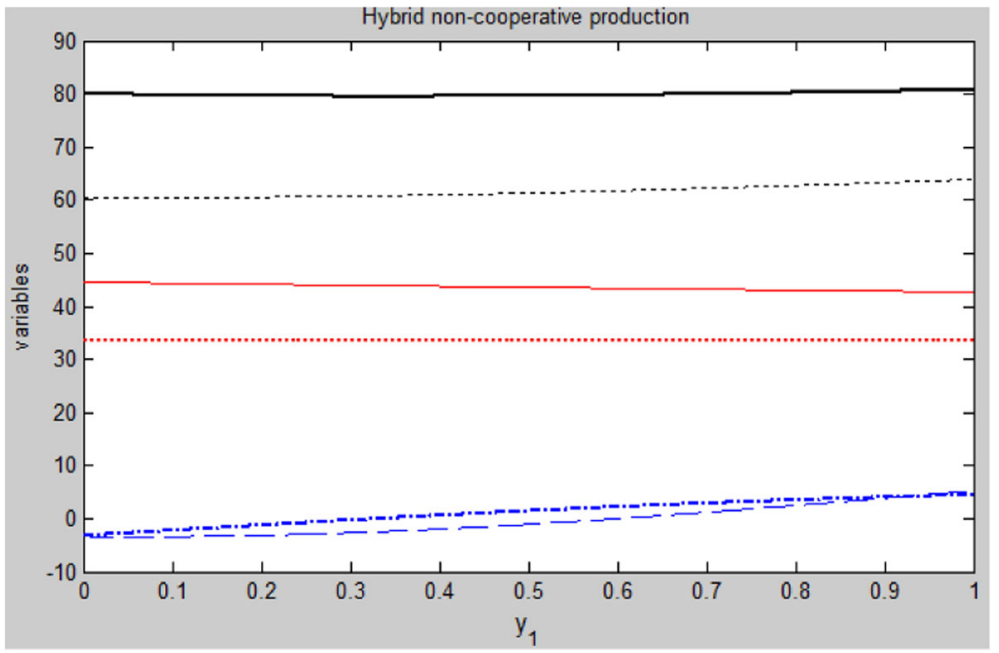

Fig. 12 The effect of $y_{1}$ on variables in non-cooperative game 


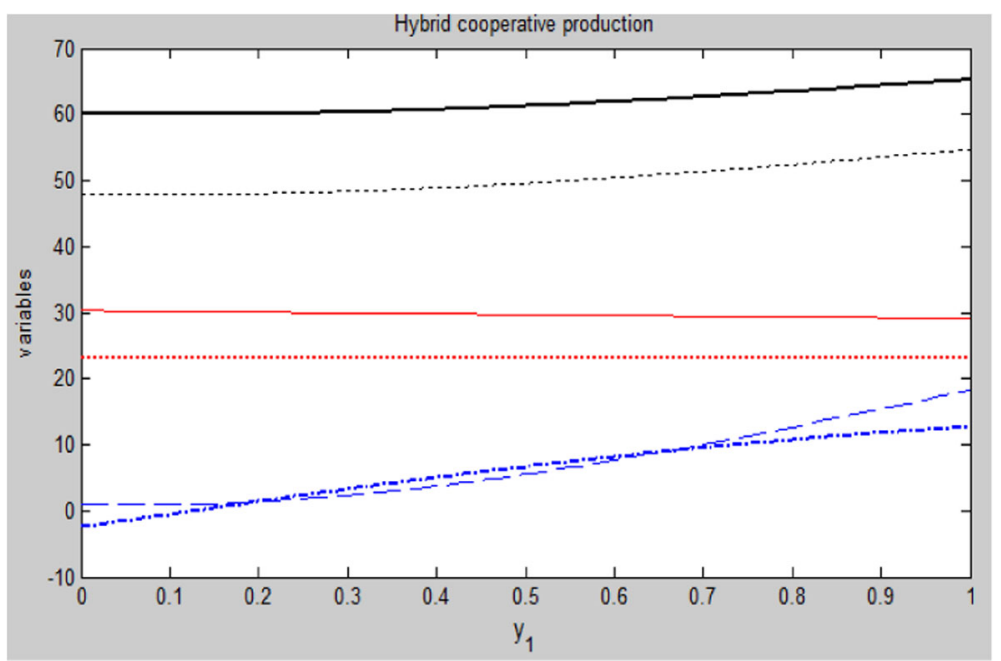

Fig. 13 The effect of $y_{1}$ on variables in cooperative game

quantity of non-green one rise. The effects of changes of $\tau_{1}$ and $\tau_{2}$ are shown in Figs. 19 and 20 and have inverse treatments on demands of green and non-green products and the pattern of changes are linear form.

$$
\longrightarrow Q_{g}^{N} \ldots \ldots \cdots \cdot Q_{g}^{C} \longrightarrow Q_{n}^{N} \cdots \cdots \cdot Q_{n}^{C}
$$

When $\tau_{1}$ increases the demand quantity of green products increase too, but the non-green products demands decrease. In the other hand, when $\tau_{2}$ increases the demand quantity of green products decreases and the non-green products demands rise. Increases of $y_{1}$ cause increases of non-green products demand and decreases the

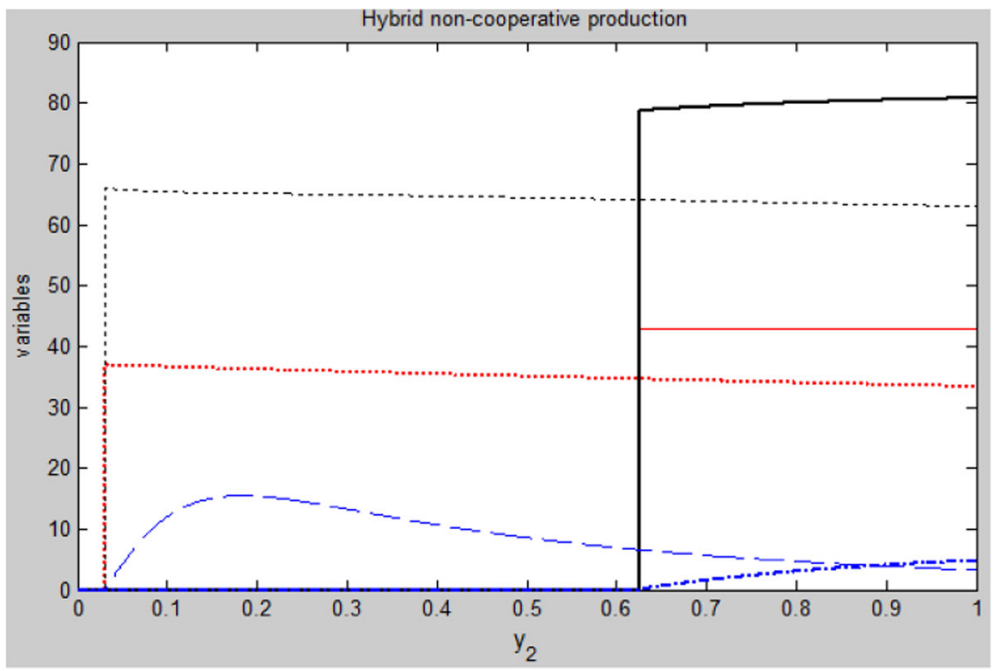

Fig. 14 The effect of $y_{2}$ on variables in non-cooperative game 


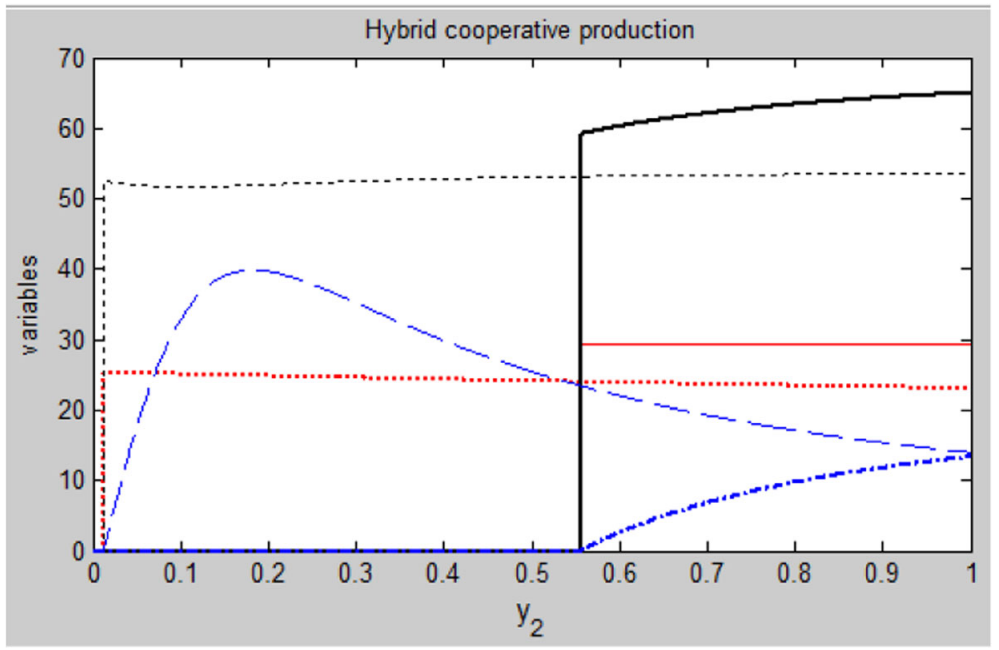

Fig. 15 The effect of $y_{2}$ on variables in cooperative game

demand of green items as presented in Fig. 21 under exponential patterns. Finally the effects of $y_{2}$ are presented in Fig. 22.

\section{Effect of parameter on profits and refund amount of products}

In this part we analyze the effect of parameters on refund amount of green and non-green products and profits of supplier, manufacturer, and system in cooperative and non-cooperative situations. From (Table 1) the profit of system in the cooperative case is more than noncooperative one.

By increasing the value of $\lambda$, refund amount of green product and profits in cooperative and non-cooperative cases are increased and refund amount of non-green product is decreased. Changes of $y_{1}, y_{2}$ have direct relations with refund amount and system profits, unless $y_{2}$ has contrariwise relationship with the refund amount of non-green products. $\varphi_{1}$ and $\tau_{1}$ have a

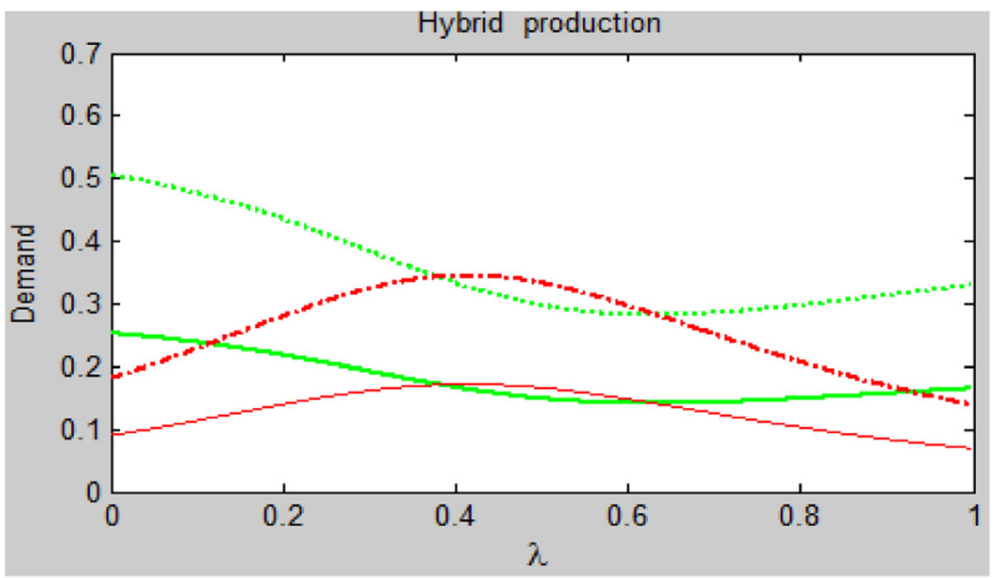

Fig. 16 Effect of $\lambda$ on demand of green and non-green product 


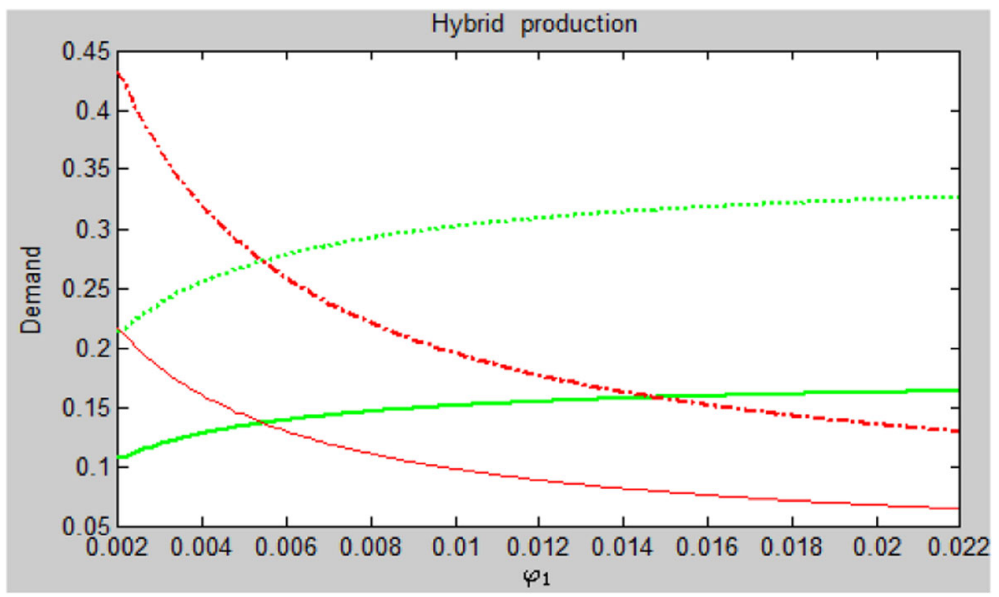

Fig. 17 Effect of $\varphi_{1}$ on demand of green and non-green product

similar behavior and by increasing them the total profit and refund amount of non-green item is reduced. But the refund amount of green product is increased. $\varphi_{2}$ and $\tau_{2}$ have a contrariwise relationship with the system profit in cooperative and non-cooperative games.

\section{Conclusion}

In the hybrid production supply chain, we propose and develop a decision model by joining the pricing strategy, refund policy, and cooperation strategy together. We develop a mathematical model for a two stages chain that contains a supplier and a manufacturer. This supply chain produces two types of products; green and non-green products such that they have a similar usage and functional but in price and environmental effects have significant differences. These products have a different raw material, so their wholesale prices and production

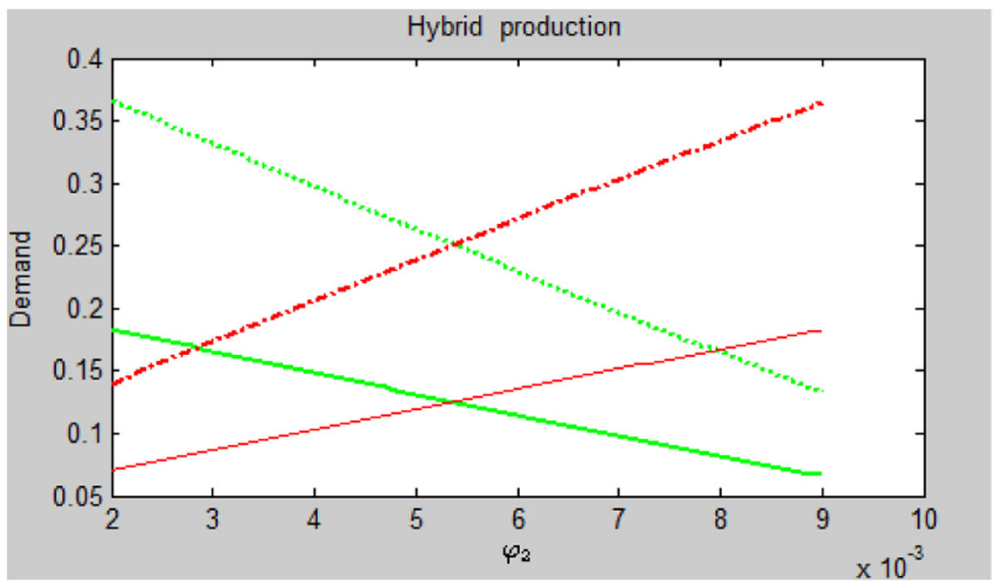

Fig. 18 Effect of $\varphi_{2}$ on demand of green and non-green product 


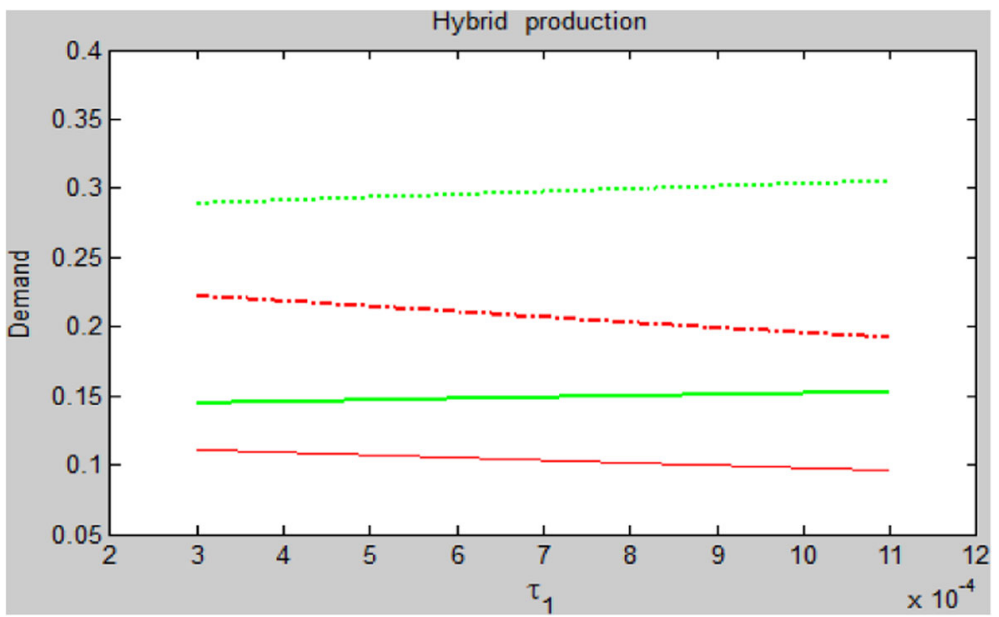

Fig. 19 Effect of $\tau_{1}$ on demands of green and non-green product

costs are different. We consider the refund policy for both products, if a customer is not satisfied from the product specifications, so he/she is permitted to return the product to the manufacture and pays back the refund amount should be determined by the manufacturer.

We explore and investigate the best decisions under cooperative and non-cooperative strategies took place between the members of chain. In non-cooperative situation, we use the Stackelberg game such that the supplier acts as a leader and the manufacture acts as a follower. Moreover in the cooperative strategy for achieving more profit, they join together. In cooperative game we use the Rubinstein bargaining game to determine the fair and reasonable raw materials whole sale prices.

We divide the results in three main parts. In the firth part we investigate on the effect of parameters on profits and decision variables using both cooperative and non-cooperative

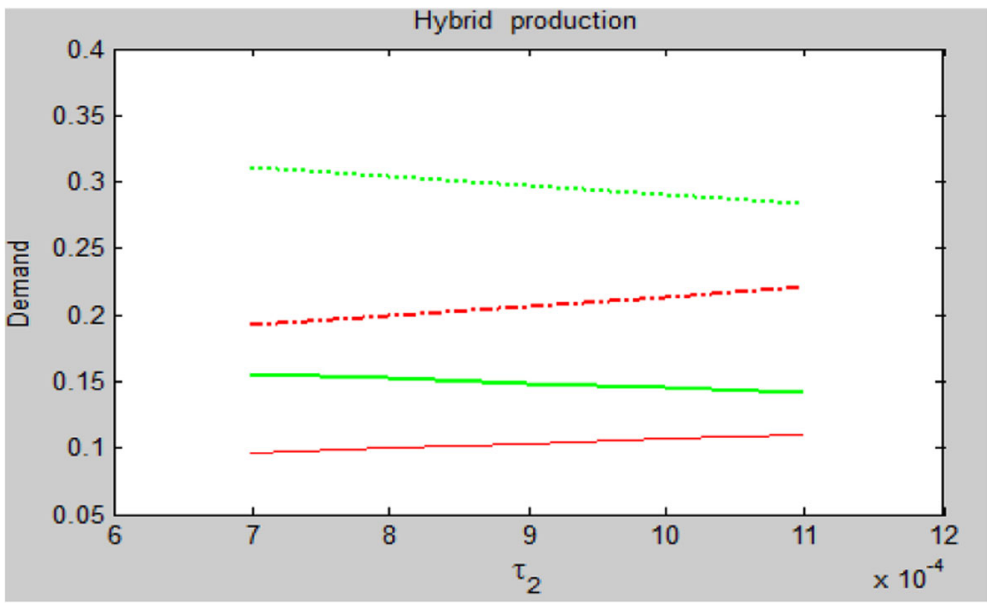

Fig. 20 Effect of $\tau_{2}$ on demand of green and non-green product 


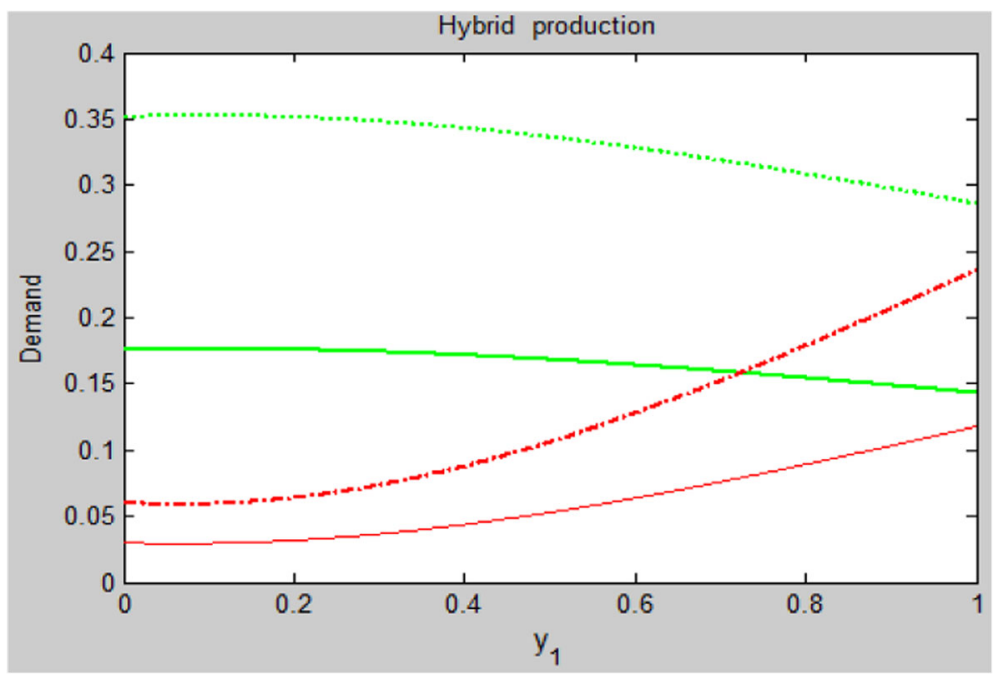

Fig. 21 Effect of $y_{1}$ on demands of green and non-green product

games. In the second part the effects of parameters on demands of green and non-green products are studied, and we observe that when cooperation happens between the supply chain members, the demands noticeably increase for both products and in the third part the profit and refund amount sensitivities of each product are investigated. Although in cooperation case, the refund amount of product is increased, but the main purpose which is increasing profit is happened.

We assumed that there is no advertising on green products from the manufacturer or government. So encouraging the customers for purchasing the green product by advertising, government aid and so on can consider as a future research. In refund

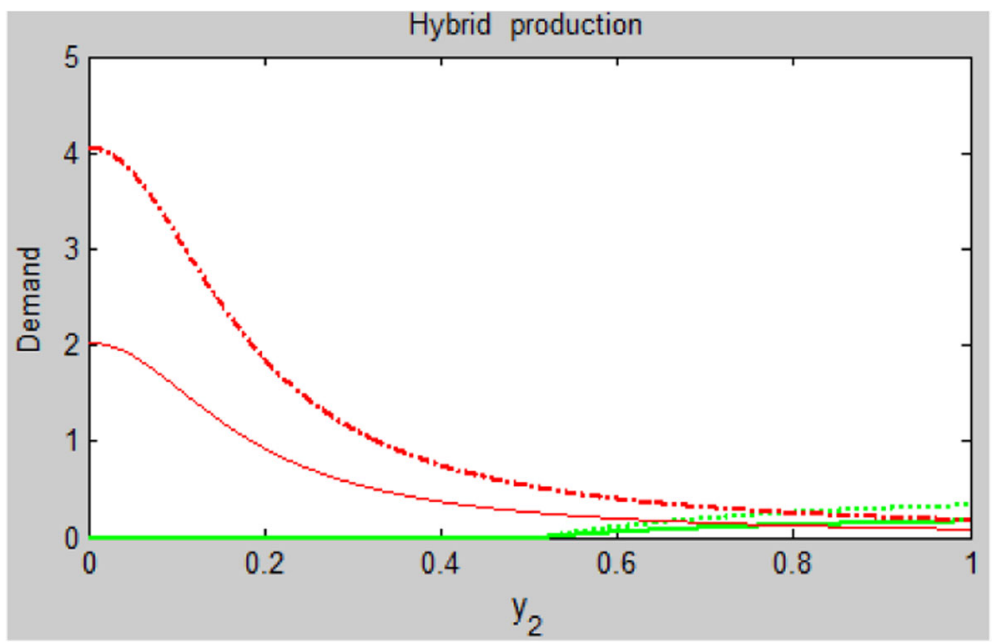

Fig. 22 Effect of $y_{2}$ on demands on green and non-green product 
Table 1 effect of parameter on system profits and refund amount in cooperative and non-cooperative game

\begin{tabular}{|c|c|c|c|c|c|c|}
\hline & & $\left(R_{g}^{C}, R_{g}{ }^{N}\right)$ & $\left(R_{n}{ }^{C}, R_{n}{ }^{N}\right)$ & $\left(\pi_{m}^{C}, \pi_{m}^{N}\right)$ & $\left(\pi_{s}^{C}, \pi_{s}^{N}\right)$ & $\left(\pi^{C}, \pi^{N}\right)$ \\
\hline \multirow[t]{5}{*}{$\lambda$} & 0.9 & $(0.161,0.099)$ & $(0.098,0.070)$ & $(6.794,4.859)$ & $(11.151,8.724)$ & $(17.945,13.583)$ \\
\hline & 0.85 & $(0.158,0.097)$ & $(0.105,0.072)$ & $(6.722,4.790)$ & $(11.063,8.663)$ & $(17.784,13.453)$ \\
\hline & 0.8 & $(0.154,0.095)$ & $(0.112,0.074)$ & $(6.646,4.720)$ & $(10.965,8.594)$ & $(17.611,13.314)$ \\
\hline & 0.75 & $(0.151,0.094)$ & $(0.120,0.077)$ & $(6.567,4.649)$ & $(10.860,8.519)$ & $(17.427,13.167)$ \\
\hline & 0.7 & $(0.149,0.093)$ & $(0.129,0.081)$ & $(6.486,4.577)$ & $(10.75,8.439)$ & $(17.236,13.016)$ \\
\hline \multirow[t]{5}{*}{$y_{1}$} & 1 & $(0.163,0.100)$ & $(0.124,0.081)$ & $(6.773,4.806)$ & $(11.184,8.768)$ & $(17.958,13.574)$ \\
\hline & 9.5 & $(0.159,0.098)$ & $(0.118,0.078)$ & $(6.709,4.763)$ & $(11.074,8.680)$ & $(17.783,13.443)$ \\
\hline & 0.9 & $(0.154,0.095)$ & $(0.112,0.074)$ & $(6.646,4.720)$ & $(10.965,8.594)$ & $(17.611,13.314)$ \\
\hline & 0.85 & $(0.149,0.093)$ & $(0.106,0.071)$ & $(6.584,4.678)$ & $(10.859,8.510)$ & $(17.443,1.188)$ \\
\hline & 0.8 & $(0.144,0.09)$ & $(0.100,0.068)$ & $(6.524,4.638)$ & $(10.755,8.427)$ & $(17.279,13.065)$ \\
\hline \multirow[t]{5}{*}{$y_{2}$} & 1 & $(0.169,0.103)$ & $(0.106,0.072)$ & $(6.734,4.781)$ & $(11.137,8.727)$ & $(17.871,13.508)$ \\
\hline & 9.5 & $(0.162,0.099)$ & $(0.109,0.073)$ & $(6.693,4.753)$ & $(11.057,8.665)$ & $(17.750,13.417)$ \\
\hline & 0.9 & $(0.154,0.095)$ & $(0.112,0.074)$ & $(6.646,4.720)$ & $(10.965,8.594)$ & $(17.611,13.314)$ \\
\hline & 0.85 & $(0.145,0.091)$ & $(0.115,0.076)$ & $(6.592,4.682)$ & $(10.859,8.513)$ & $(17.451,13.195)$ \\
\hline & 0.8 & $(0.135,0.086)$ & $(0.119,0.078)$ & $(6.529,4.638)$ & $(10.736,8.418)$ & $(17.265,13.056)$ \\
\hline \multirow[t]{5}{*}{$\varphi_{1}\left(10^{-3}\right)$} & 10 & $(0.156,0.096)$ & $(0.107,0.072)$ & $(6.592,4.678)$ & $(10.892,8.537)$ & $(17.483,13.215)$ \\
\hline & 9.5 & $(0.155,0.096)$ & $(0.109,0.073)$ & $(6.618,4.698)$ & $(10.927,8.564)$ & $(17.544,13.262)$ \\
\hline & 9 & $(0.154,0.095)$ & $(0.112,0.074)$ & $(6.646,4.720)$ & $(10.965,8.594)$ & $(17.611,13.314)$ \\
\hline & 8.5 & $(0.153,0.095)$ & $(0.115,0.076)$ & $(6.677,4.744)$ & $(11.007,8.627)$ & $(17.684,13.370)$ \\
\hline & 8 & $(0.152,0.094)$ & $(0.118,0.070)$ & $(6.710,4.770)$ & $(11.053,8.662)$ & $(17.763,13.432)$ \\
\hline \multirow{5}{*}{$\varphi_{2}\left(10^{-3}\right)$} & 5 & $(0.139,0.088)$ & $(0.127,0.082)$ & $(6.540,4.624)$ & $(10.835,8.501)$ & $(17.185,13.125)$ \\
\hline & 4.5 & $(0.147,0.092)$ & $(0.119,0.078)$ & $(6.592,4.670)$ & $(10.901,8.548)$ & $(17.075,13.219)$ \\
\hline & 4 & $(0.154,0.095)$ & $(0.112,0.074)$ & $(6.646,4.720)$ & $(10.965,8.594)$ & $(17.611,13.314)$ \\
\hline & 3.5 & $(0.162,0.099)$ & $(0.104,0.071)$ & $(6.702,4.775)$ & $(11.028,8.637)$ & $(16.960,13.412)$ \\
\hline & 3 & $(0.169,0.103)$ & $(0.097,0.067)$ & $(6.762,4.836)$ & $(11.089,8.677)$ & $(16.723,13.513)$ \\
\hline \multirow[t]{5}{*}{$\tau_{1}\left(10^{-4}\right)$} & 8 & $(0.158,0.100)$ & $(0.110,0.074)$ & $(6.395,4.730)$ & $(10.891,8.537)$ & $(17.536,13.268)$ \\
\hline & 7.5 & $(0.156,0.097)$ & $(0.111,0.074)$ & $(6.642,4.725)$ & $(10.931,8.566)$ & $(17.573,13.290)$ \\
\hline & 7 & $(0.154,0.095)$ & $(0.112,0.074)$ & $(6.646,4.720)$ & $(10.965,8.594)$ & $(17.611,13.314)$ \\
\hline & 6.5 & $(0.152,0.093)$ & $(0.113,0.075)$ & $(6.651,4.716)$ & $(10.999,8.622)$ & $(17.611,13.338)$ \\
\hline & 6 & $(0.150,0.091)$ & $(0.114,0.075)$ & $(6.656,4.713)$ & $(11.032,8.650)$ & $(17.649,13.363)$ \\
\hline \multirow[t]{5}{*}{$\tau_{2}\left(10^{-4}\right)$} & 10 & $(0.151,0.094)$ & $(0.117,0.079)$ & $(6.645,4.743)$ & $(10.883,8.524)$ & $(17.529,13.267)$ \\
\hline & 9.5 & $(0.153,0.095)$ & $(0.114,0.077)$ & $(6.645,4.731)$ & $(10.925,8.559)$ & $(17.570,13.290)$ \\
\hline & 9 & $(0.154,0.095)$ & $(0.112,0.074)$ & $(6.646,4.720)$ & $(10.965,8.594)$ & $(17.611,13.314)$ \\
\hline & 8.5 & $(0.156,0.096)$ & $(0.109,0.072)$ & $(6.647,4.710)$ & $(11.005,8.628)$ & $(17.652,13.338)$ \\
\hline & 8 & $(0.157,0.097)$ & $(0.107,0.070)$ & $(6.649,4.700)$ & $(11.044,8.662)$ & $(17.693,13.363)$ \\
\hline
\end{tabular}

policy, we consider the refund amount and the selling price of product, while product quality level, one of the most important factors to avoid return, is not mentioned. So considering quality level as the third factor affecting the both demand and return quantities could be of interests.

\section{Appendix A}

\section{Proof of Theorem 1}

In order to prove that the profit function of manufacturer in non-cooperative strategy shown in Eq. (7) is concave and also has an optimal solution, in the first step we derive the first order derivatives of profit function with respect to decision variables. So we have; 


$$
\begin{aligned}
& \frac{\delta \pi_{m}^{N}}{\delta P_{g}}=1+\frac{1}{A(1-\lambda)}\left(-2 P_{g}+2 P_{n}+y_{1} r_{g}-y_{2} r_{n}+w_{1}+c_{R}-w_{0}-c_{r}\right)-\tau_{1} r_{g} \\
& \frac{\delta \pi_{m}^{N}}{\delta P_{n}}=\frac{1}{A(1-\lambda) \lambda}\left(2 \lambda P_{g}-2 P_{n}-\lambda y_{1} r_{g}+y_{2} r_{n}-\lambda w_{1}-\lambda c_{R}+w_{0}+c_{r}\right)-\tau_{2} r_{n} \\
& \frac{\delta \pi_{m}^{N}}{\delta r_{g}}=\frac{y_{1}}{A(1-\lambda)}\left(P_{g}-P_{n}-w_{1}-c_{R}+w_{0}+c_{r}\right)-\phi_{1}-2 \varphi_{1} r_{g}-\tau_{1} P_{g} \\
& \frac{\delta \pi_{m}^{N}}{\delta r_{n}}=\frac{y_{2}}{A(1-\lambda)}\left(-P_{g}+w_{1}+c_{R}\right)+\frac{y_{2}}{A \lambda(1-\lambda)}\left(P_{n}-w_{0}-c_{r}\right)-\phi_{2}-2 \varphi_{2} r_{n}-\tau_{2} P_{n}
\end{aligned}
$$

And the second order derivatives are,

$$
\begin{gathered}
\frac{\partial^{2} \pi}{\partial P_{g}{ }^{2}}=\frac{-2}{A(1-\lambda)}, \frac{\partial^{2} \pi}{\partial P_{n}{ }^{2}}=\frac{-2}{A \lambda(1-\lambda)}, \frac{\partial^{2} \pi}{\partial r_{g}{ }^{2}}=-2 \varphi_{1}, \frac{\partial^{2} \pi}{\partial r_{n}{ }^{2}}=-2 \varphi_{2}, \frac{\partial^{2} \pi}{\partial P_{g} \partial P_{n}}=\frac{2}{A(1-\lambda)}, \frac{\partial^{2} \pi}{\partial r_{g} \partial r_{n}}=0 \\
\frac{\partial^{2} \pi}{\partial P_{g} \partial r_{g}}=\frac{y_{1}}{A(1-\lambda)}-\tau_{1}, \frac{\partial^{2} \pi}{\partial P_{g} \partial r_{n}}=\frac{-y_{2}}{A(1-\lambda)}-\tau_{1}, \frac{\partial^{2} \pi}{\partial P_{n} \partial r_{g}}=\frac{-y_{1}}{A(1-\lambda)}, \frac{\partial^{2} \pi}{\partial P_{n} \partial r_{n}}=\frac{y_{2}}{A \lambda(1-\lambda)}-\tau_{2},
\end{gathered}
$$

Then the hessian matrix of the manufacture's profit function for hybrid production mode is;

$$
H=\left(\frac{1}{A(1-\lambda)}\right)\left[\begin{array}{cccc}
-2 & y_{1}-\tau_{1} A(1-\lambda) & 2 & -y_{2}-\tau_{1} A(1-\lambda) \\
y_{1}-\tau_{1} A(1-\lambda) & -2 \varphi_{1} A(1-\lambda) & -y_{1} & 0 \\
2 & -y_{1} & \frac{-2}{\lambda} & \frac{y_{2}}{\lambda}-\tau_{2} A(1-\lambda) \\
-y_{2}-\tau_{1} A(1-\lambda) & 0 & \frac{y_{2}}{\lambda}-\tau_{2} A(1-\lambda) & -2 \varphi_{2} A(1-\lambda)
\end{array}\right]
$$

To ensure that manufacturing have optimal solution the hessian matrix must be negative and so must meet the following conditions,

$$
\begin{gathered}
H_{1}=-2<0, H_{2}=\left|\begin{array}{ccc}
-2 & y_{1}-\tau_{1} A(1-\lambda) \\
y_{1}-\tau_{1} A(1-\lambda) & -2 \varphi_{1} A(1-\lambda)
\end{array}\right|>0, \\
H_{3}=\left|\begin{array}{ccc}
-2 & y_{1}-\tau_{1} A(1-\lambda) & 2 \\
y_{1}-\tau_{1} A(1-\lambda) & -2 \varphi_{1} A(1-\lambda) & -y_{1} \\
2 & -y_{1} & \frac{-2}{\lambda}
\end{array}\right|<0 \\
H_{4}=\left|\begin{array}{cccc}
-2 & y_{1}-\tau_{1} A(1-\lambda) & 2 & -y_{2}-\tau_{1} A(1-\lambda) \\
y_{1}-\tau_{1} A(1-\lambda) & -2 \varphi_{1} A(1-\lambda) & -y_{1} & 0 \\
2 & -y_{1} & \frac{-2}{\lambda} & \frac{y_{2}}{\lambda}-\tau_{2} A(1-\lambda) \\
-y_{2}-\tau_{1} A(1-\lambda) & 0 & \frac{y_{2}}{\lambda}-\tau_{2} A(1-\lambda) & -2 \varphi_{2} A(1-\lambda)
\end{array}\right|>0
\end{gathered}
$$

It should be noted that in the above conditions are not met, using optimization software one can find the optimal values of decision variables.

\section{Appendix B}

\section{Proof of Theorem 2}

In order to prove that the profit function of non-cooperative case shown in Eq. (15) is concave and also has an optimal solution, in the first step we derive the first order derivatives of profit function with respect to decision variables. So we have; 


$$
\begin{aligned}
& \frac{\delta \pi^{C}}{\delta P_{g}}=1+\frac{1}{A(1-\lambda)}\left(-2 P_{g}+2 P_{n}+y_{1} r_{g}-y_{2} r_{n}+c_{R}-c_{r}\right)-\tau_{1} r_{g} \\
& \frac{\delta \pi^{C}}{\delta P_{n}}=\frac{1}{A(1-\lambda) \lambda}\left(2 \lambda P_{g}-2 P_{n}-\lambda y_{1} r_{g}+y_{2} r_{n}-\lambda c_{s}-\lambda c_{R}+c_{s}+c_{r}\right)-\tau_{2} r_{n} \\
& \frac{\delta \pi^{C}}{\delta r_{g}}=\frac{y_{1}}{A(1-\lambda)}\left(P_{g}-P_{n}-c_{R}+c_{r}\right)-\phi_{1}-2 \varphi_{1} r_{g}-\tau_{1} P_{g} \\
& \frac{\delta \pi^{C}}{\delta r_{n}}=\frac{y_{2}}{A(1-\lambda)}\left(-P_{g}+c_{s}+c_{R}\right)+\frac{y_{2}}{A \lambda(1-\lambda)}\left(P_{n}-c_{s}-c_{r}\right)-\phi_{2}-2 \varphi_{2} r_{n}-\tau_{2} P_{n}
\end{aligned}
$$

So the second order derivatives are,

$$
\begin{gathered}
\frac{\partial^{2} \pi}{\partial P_{g}{ }^{2}}=\frac{-2}{A(1-\lambda)}, \frac{\partial^{2} \pi}{\partial P_{n}{ }^{2}}=\frac{-2}{A \lambda(1-\lambda)}, \frac{\partial^{2} \pi}{\partial r_{g}{ }^{2}}=-2 \varphi_{1}, \frac{\partial^{2} \pi}{\partial P_{g} \partial P_{n}}=\frac{2}{A(1-\lambda)}, \frac{\partial^{2} \pi}{\partial P_{g} \partial r_{g}}=\frac{y_{1}}{A(1-\lambda)}-\tau_{1}, \\
\frac{\partial^{2} \pi}{\partial P_{g} \partial r_{n}}=\frac{-y_{2}}{A(1-\lambda)}-\tau_{1}, \frac{\partial^{2} \pi}{\partial P_{n} \partial r_{g}}=\frac{-y_{1}}{A(1-\lambda)}, \frac{\partial^{2} \pi}{\partial r_{g} \partial r_{n}}=0 \frac{\partial^{2} \pi}{\partial P_{n} \partial r_{n}}=\frac{y_{2}}{A \lambda(1-\lambda)}-\tau_{2}, \frac{\partial^{2} \pi}{\partial r_{n}{ }^{2}}=-2 \varphi_{2},
\end{gathered}
$$

Then the hessian matrix of the profit for hybrid production mode is;

$$
H=\left(\frac{1}{A(1-\lambda)}\right)\left[\begin{array}{cccc}
-2 & y_{1}-\tau_{1} A(1-\lambda) & 2 & -y_{2}-\tau_{1} A(1-\lambda) \\
y_{1}-\tau_{1} A(1-\lambda) & -2 \varphi_{1} A(1-\lambda) & -y_{1} & 0 \\
2 & -y_{1} & \frac{-2}{\lambda} & \frac{y_{2}}{\lambda}-\tau_{2} A(1-\lambda) \\
-y_{2}-\tau_{1} A(1-\lambda) & 0 & \frac{y_{2}}{\lambda}-\tau_{2} A(1-\lambda) & -2 \varphi_{2} A(1-\lambda)
\end{array}\right]
$$

To ensure that manufacturing have optimal solution the hessian matrix must be negative, so must meet this condition,

$$
\begin{aligned}
H_{1} & =-2<0, \quad H_{2}=\left|\begin{array}{cc}
-2 & y_{1}-\tau_{1} A(1-\lambda) \\
y_{1}-\tau_{1} A(1-\lambda) & -2 \varphi_{1} A(1-\lambda)
\end{array}\right|>0, \\
H_{3} & =\left|\begin{array}{ccc}
-2 & y_{1}-\tau_{1} A(1-\lambda) & 2 \\
y_{1}-\tau_{1} A(1-\lambda) & -2 \varphi_{1} A(1-\lambda) & -y_{1} \\
2 & -y_{1} & \frac{-2}{\lambda}
\end{array}\right|<0, \\
H_{4} & =\left|\begin{array}{cccc}
y_{1}-\tau_{1} A(1-\lambda) & -2 \varphi_{1} A(1-\lambda) & -y_{1} & -y_{2}-\tau_{1} A(1-\lambda) \\
2 & -y_{1} & \frac{-2}{\lambda} & \frac{y_{2}}{\lambda}-\tau_{2} A(1-\lambda) \\
-y_{2}-\tau_{1} A(1-\lambda) & 0 & \frac{y_{2}}{\lambda}-\tau_{2} A(1-\lambda) & -2 \varphi_{2} A(1-\lambda)
\end{array}\right|>0
\end{aligned}
$$

It should be noted that in the above conditions are not met, using optimization software one can find the optimal values of decision variables.

\section{References}

1. Guide VDR, Wassenhove LN (2006a) Closed-loop supply chains: an Introduction to the feature issue (part 1). Prod Oper Manag 15(3):345-350

2. Guide VDR, Van Wassenhove LN (2006b) Closed-loop supply chains: an Introduction to the feature issue (part 2). Prod Oper Manag 15(4):471-472 
3. Hall J, Vredenburg H (2012) The challenges of innovating for sustainable development. MIT Sloan Management Review. MIT press

4. Klassen RD, McLaughlin CP (1996) The impact of environmental management on firm performance. Manag Sci 42(8):1199-1214

5. Kumar S, Putnam V (2008) Cradle to cradle: reverse logistics strategies and opportunities across three industry sectors. Int J Prod Econ 115(2):305-315

6. O’Brien C (1999) Sustainable production - a new paradigm for a new millennium. Int J Prod Econ 60:1-7

7. Östlin J, Sundin E, Björkman M (2008) Importance of closed-loop supply chain relationships for product remanufacturing. Int J Prod Econ 115(2):336-348

8. Sroufe R (2003) Effectsofenvironmentalmanagementsystemsonenvironmental management practicesandoperations. Prod Oper Manag 12(3):416-431

9. Zhang C-T, Wang H-X, Ren M-L (2014) Research on pricing and coordination strategy of green supply chain under hybrid production mode. Comput Ind Eng 72:24-31

10. Balachander S (2001) Warranty Signalling and reputation. Manag Sci 47(9):1282-1289

11. Li Y, Xu L, Li D (2013) Examining relationships between the return policy, product quality, and pricing strategy in online direct selling. Int J Prod Econ 144(2):451-460

12. Wood SL (2001) Remote purchase environments: the influence of return policy leniency on two-stage decision processes. J Mark Res 38(2):157-169

13. Mukhopadhyay SK, Setaputra R (2007) A dynamic model for optimal design quality and return policies. Eur J Oper Res 180(3):1144-1154

14. Navinchandra D (1990) Steps toward Environmentally Compatible. CARNEGIE-MELLON UNIV PITTSBURGH PA ROBOTICS INST, No. CMU-RI

15. Vachon S, Klassen RD (2008) Environmental management and manufacturing performance: the role of collaboration in the supply chain. Int J Prod Econ 111(2):299-315

16. Chen YJ, Sheu J-B (2009) Environmental-regulation pricing strategies for green supply chain management. Transport Res E-Log 45(5):667-677

17. Ghosh D, Shah J (2012) A comparative analysis of greening policies across supply chain structures. Int J Prod Econ 135(2):568-583

18. Zhang CT, Liu LP (2013) Research on coordination mechanism in three-level green supply chain under non-cooperative game. Appl Math Model 37(5):3369-3379

19. Li B, Zhu M, Jiang Y, Li Z (2015) Pricing policies of a competitive dual-channel green supply chain. J Clean Prod:1-14

20. Giri BC, Bardhan S (2016) Coordinating a two-echelon supply chain with environmentally aware consumers. Int J Manag Sci Eng Manag 11(3):178-185

21. Rao P, Holt D (2005) Do green supply chains lead to competitiveness and economic performance? Int J Oper Prod Manag 25(9):898-916

22. Chen Y-S, Lai S-B, Wen C-T (2006) The influence of green innovation performance on corporate advantage in Taiwan. J Bus Ethics 67(4):331-339

23. Chiou T-Y, Chan HK, Lettice F, Chung SH (2011) The influence of greening the suppliers and green innovation on environmental performance and competitive advantage in Taiwan. Transport Res E-Log 47(6):822-836

24. Bowen FE, Cousine PD, Lamming RC, Faruk A (2001) Explaining the gap between the theory and practice of green supply. Greener Management International 35:41-59

25. Min H, Galle WP (1997) Green purchasing strategies: trends and implications. J Supply Chain Manag 33(3):10-17

26. Olugu EU, Wong KY, Shaharoun AM (2011) Development of key performance measures for the automobile green supply chain. Resour Conserv Recycl 55(6):567-579

27. Liang L, Yang F, Cook WD, Zhu J (2006) DEA models for supply chain efficiency evaluation. Ann Oper Res 145(1):35-49

28. Cao J, Zhang X (2013) Coordination strategy of green supply chain under the free market mechanism. Energy Procedia 36:1130-1137

29. Sheu J-B (2011) Bargaining framework for competitive green supply chains under governmental financial intervention. Transport Res E-Log 47(5):573-592

30. Webster S, Weng ZK (2000) A risk-free perishable item returns policy. Manufacturing \& Service Operations Management 2(1):100-106

31. Taylor T (2002) Supply chain coordination under channel rebates with sales effort effects. Manag Sci 48(8): 992-1007

32. Yue X, Raghunathan S (2007) The impacts of the full returns policy on a supply chain with information asymmetry. Eur J Oper Res 180(2):630-647 
33. Yao Z, Leung SCH, Lai KK (2008) Analysis of the impact of price-sensitivity factors on the returns policy in coordinating supply chain. Eur J Oper Res 187(1):275-282

34. He Y, Zhao X, Zhao L, He J (2009) Coordinating a supply chain with effort and price dependent stochastic demand. Appl Math Model 33(6):2777-2790

35. Xiao T, Shi K, Yang D (2010) Coordination of a supply chain with consumer return under demand uncertainty. Int J Prod Econ 124(1):171-180

36. Liu J, Mantin B, Wang H (2014) Supply chain coordination with customer returns and refund-dependent demand. Int J Prod Econ 148:81-89

37. Yoo SH (2014) Product quality and return policy in a supply chain under risk aversion of a supplier. Int J Prod Econ 154:146-155

38. Taleizadeh AA, Niaki ST, Aryanezhad MB (2008a) Multi-product multi-Constraint inventory control systems with stochastic replenishment and discount under Fuzzy purchasing price and holding costs. Am J Appl Sci 8(7):1228-1234

39. Taleizadeh AA, Aryanezhad MB, Niaki STA (2008b) Optimizing multi-products multi-constraints inventory control systems with stochastic replenishments. J Appl Sci 6(1):1-1

40. Taleizadeh AA, Moghadasi H, Niaki STA, Eftekhari AK (2009) An EOQ-joint replenishment policy to supply expensive imported raw materials with payment in advance. J Appl Sci 8(23):4263-4273

41. Taleizadeh AA, Niaki STA, Aryanezhad MB (2010a) Replenish-up-to multi chance-Constraint inventory control system with stochastic period lengths and Total discount under Fuzzy purchasing price and holding costs. Int J Syst Sci 41(10):1187-1200

42. Taleizadeh AA, Niaki STA, Aryanezhad MB, Fallah-Tafti A (2010b) A genetic algorithm to optimize multiproduct multi-Constraint inventory control systems with stochastic replenishments and discount. Int J Adv Manuf Technol 51(1-4):311-323

43. Taleizadeh AA, Barzinpour F, Wee HM (2011) Meta-heuristic algorithms to solve the Fuzzy single period problem. Math Comput Model 54(5-6):1273-1285

44. Taleizadeh AA, Pentico DW, Aryanezhad MB, Ghoreyshi M (2012) An economic order quantity model with partial Backordering and a special sale price. Eur J Oper Res 221(3):571-583

45. Taleizadeh AA, Pentico DW, Jabalameli MS, Aryanezhad MB (2013a) An economic order quantity model with multiple partial prepayments and partial Backordering. Math Comput Model 57(3-4):311-323

46. Taleizadeh AA, Pentico DW (2013b) An economic order quantity model with known price increase and partial Backordering. Eur J Oper Res 28(3):516-525

47. Taleizadeh AA, Wee HM, Jalali-Naini SGR (2013b) Economic production quantity model with repair failure and limited capacity. Appl Math Model 37(5):2765-2774

48. Taleizadeh AA (2014a) An economic order quantity model with partial Backordering and advance payments for an evaporating item. Int J Prod Econ 155:185-193

49. Taleizadeh AA, Cardenas-Barron LE, Mohammadi B (2014b) Multi product single machine EPQ model with Backordering, scraped products, rework and interruption in manufacturing process. Int J Prod Econ 150:9-27

50. Taleizadeh AA, Nematollahi MR (2014c) An inventory control problem for deteriorating items with Backordering and financial Engineering considerations. Appl Math Model 38:93-109

51. Taleizadeh AA, Pentico DW (2014d) An economic order quantity model with partial Backordering and allunits discount. Int J Prod Econ 155:172-184

52. Mukhopadhyay SK, Setoputro R (2004) Reverse logistics in e-business. Int J Phys Distrib Logist Manag 34(1):70-89

53. Ringbom S, Shy O (2004) Advance booking, cancellations, and partial refunds. Econ Bull 13(1):1-7

54. Ding D, Chen J (2008) Coordinating a three level supply chain with flexible return policies. Omega 36(5): $865-876$

55. Su X (2009) Consumer returns policies and supply chain performance. Manufacturing \& Service Operations Management 11(4):595-612

56. Xu L, Li Y, Govindan K, Xu X (2015) Consumer returns policies with endogenous deadline and supply chain coordination. Eur J Oper Res 242(1):88-99

57. Roy A, Sana SS, Chaudhuri K (2015) Effect of cooperative advertising policy for two layer supply chain. International Journal of Management Science and Engineering Management 10(2):89-101

58. Chen J (2011) Returns with wholesale-price-discount contract in a newsvendor problem. Int J Prod Econ 130(1):104-111

59. He J, Chin KS, Yang JB, Zhu DL (2006) Return policy model of supply chain management for singleperiod products. J Optim Theory Appl 129(2):293-308

60. Majumder P, Srinivasan A (2008) Leadership and competition in network supply chains. Manag Sci 54(6): 1189-1204

61. Choi SC (1991) Price competition in a channel structure with a common retailer. Mark Sci 10(4):271-296 
62. Choi SC (1996) Price competition in a duopoly common retailer channel. J Retail 72(2):117-134

63. Choi TM, Li Y, Xu L (2013) Channel leadership, performance and coordination in closed loop supply chains. Int J Prod Econ 146(1):371-380

64. Lee E, Staelin R (1997) Vertical strategic interaction: implications for channel pricing strategy. Mark Sci 16(3):185-207

65. Almehdawe E, Matin B (2010) Vendor managed inventory with a capacitated manufacturer and multiple retailer: retailer versus manufacturer leadership. Int J Prod Econ 128(1):292-302

66. Gao J, Han H, Hou L, Wang H (2016) Pricing and effort decisions in a closed-loop supply chain under different channel power structures. J Clean Prod 112:2043-2057

67. Xiao T, Choi TM, Cheng TE (2014) Product variety and channel structure strategy for a retailer-Stackelberg supply chain. Eur J Oper Res 233(1):114-124

68. Xu A, Hu X, Gao S 2011 Game model between enterprises and consumers in green supply chain of home appliance industry. In: Distributed Computing and Applications to Business, Engineering and Science (DCABES), 2011 Tenth International Symposium on. IEEE, pp 96-99

69. Barari S, Agarwal G, Zhang WC, Mahanty B, Tiwari MK (2012) A decision framework for the analysis of green supply chain contracts: an evolutionary game approach. Expert Syst Appl 39(3):2965-2976

70. Moriarty RT, Moran U (1990) Managing hybrid marketing systems. Harv Bus Rev 68(6):146-155

71. Chiang WYK, Chhajed D, Hess JD (2003) Direct marketing, indirect profits: a strategic analysis of dualchannel supply-chain design. Manag Sci 49(1):1-20

72. Tsay AA, Agrawal N (2004) Channel conflict and coordination in the e-commerce age. Prod Oper Manag 13(1):93-110

73. Yao DQ, Liu JJ (2005) Competitive pricing of mixed retail and e-tail distribution channels. Omega 33(3): 235-247

74. Fruchter GE, Tapiero CS (2005) Dynamic online and offline channel pricing for heterogeneous customers in virtual acceptance. Int Game Theory Rev 7(02):137-150

75. Cattani K, Gilland W, Heese HS, Swaminathan J (2006) Boiling frogs: pricing strategies for a manufacturer adding a direct channel that competes with the traditional channel. Prod Oper Manag 15(1):40

76. Huang W, Swaminathan JM (2009) Introduction of a second channel: implications for pricing and profits. Eur J Oper Res 194(1):258-279

77. Dan B, Xu G, Liu C (2012) Pricing policies in a dual-channel supply chain with retail services. Int J Prod Econ 139(1):312-320

78. Martín-Herran G, Taboubi S (2015) Price coordination in distribution channels: a dynamic perspective. Eur J Oper Res 240(2):401e414

79. Ferrer G, Swaminathan JM (2006) Managing new and remanufactured products. Manag Sci 52:15-26

80. Rubinstein BYA (1982) Perfect equilibrium in a bargaining model. Econometrica. Journal of the Econometric Society 50:97-109 